\title{
An Empirically Based System for Processing Definite Descriptions
}

\author{
Renata Vieira* \\ Universidade do Vale do Rio dos Sinos
}

\author{
Massimo Poesio ${ }^{\dagger}$ \\ University of Edinburgh
}

We present an implemented system for processing definite descriptions in arbitrary domains. The design of the system is based on the results of a corpus analysis previously reported, which highlighted the prevalence of discourse-new descriptions in newspaper corpora. The annotated corpus was used to extensively evaluate the proposed techniques for matching definite descriptions with their antecedents, discourse segmentation, recognizing discourse-new descriptions, and suggesting anchors for bridging descriptions.

\section{Introduction}

Most models of definite description processing proposed in the literature tend to emphasise the anaphoric role of these elements. ${ }^{1}$ (Heim [1982] is perhaps the best formalization of this type of theory). This approach is challenged by the results of experiments we reported previously (Poesio and Vieira 1998), in which subjects were asked to classify the uses of definite descriptions in Wall Street Journal articles according to schemes derived from proposals by Hawkins (1978) and Prince (1981). The results of these experiments indicated that definite descriptions are not primarily anaphoric; about half of the time they are used to introduce a new entity in the discourse. In this paper, we present an implemented system for processing definite descriptions based on the results of that earlier study. In our system, techniques for recognizing discourse-new descriptions play a role as important as techniques for identifying the antecedent of anaphoric ones.

A central characteristic of the work described here is that we intended from the start to develop a system whose performance could be evaluated using the texts annotated in the experiments mentioned above. Assessing the performance of an NLP system on a large number of examples is increasingly seen as a much more thorough evaluation of its performance than trying to come up with counterexamples; it is considered essential for language engineering applications. These advantages are thought by many to offset some of the obvious disadvantages of this way of developing NLP theories-in particular, the fact that, given the current state of language processing technology, many hypotheses of interest cannot be tested yet (see below). As a result, quantitative evaluation is now commonplace in areas of language engineering such as parsing, and quantitative evaluation techniques are being proposed for semantic

\footnotetext{
* Universidade do Vale do Rio dos Sinos - UNISINOS, Av. Unisinos 950 - Cx. Postal 275, 93022-000 São Leopoldo RS Brazil. E-mail: renata@exatas.unisinos.br

$\dagger$ University of Edinburgh, ICCS and Informatics, 2, Buccleuch Place, EH8 9LW Edinburgh UK. E-mail: Massimo.Poesio@ed.ac.uk

1 We use the term definite description (Russell 1905) to indicate definite noun phrases with the definite article the, such as the car. We are not concerned with other types of definite noun phrases such as pronouns, demonstratives, or possessive descriptions. Anaphoric expressions are those linguistic expressions used to signal, evoke, or refer to previously mentioned entities.
} 
interpretation as well, for example, at the Sixth and Seventh Message Understanding Conferences (MUC-6 and MUC-7) (Sundheim 1995; Chinchor 1997), which also included evaluations of systems on the so-called coreference task, a subtask of which is the resolution of definite descriptions. The system we present was developed to be evaluated in a quantitative fashion, as well, but because of the problems concerning agreement between annotators observed in our previous study, we evaluated the system both by measuring precision/recall against a "gold standard," as done in MUC, and by measuring agreement between the annotations produced by the system and those proposed by the annotators.

The decision to develop a system that could be quantitatively evaluated on a large number of examples resulted in an important constraint: we could not make use of inference mechanisms such as those assumed by traditional computational theories of definite description resolution (e.g., Sidner 1979; Carter 1987; Alshawi 1990; Poesio 1993). Too many facts and axioms would have to be encoded by hand for theories of this type to be tested even on a medium-sized corpus. Our system, therefore, is based on a shallow-processing approach more radical even than that attempted by the first advocate of this approach, Carter (1987), or by the systems that participated in the MUC evaluations (Appelt et al. 1995; Gaizaukas et al. 1995; Humphreys et al. 1988), since we made no attempt to fine-tune the system to maximize performance on a particular domain. The system relies only on structural information, on the information provided by preexisting lexical sources such as WordNet (Fellbaum 1998), on minimal amounts of general hand-coded information, or on information that could be acquired automatically from a corpus. As a result, the system does not really have the resources to correctly resolve those definite descriptions whose interpretation does require complex reasoning (we grouped these in what we call the "bridging" class). We nevertheless developed heuristic techniques for processing these types of definites as well, the idea being that these heuristics may provide a baseline against which the gains in performance due to the use of commonsense knowledge can be assessed more clearly. $^{2}$

The paper is organized as follows: We first summarize the results of our previous corpus study (Poesio and Vieira 1998) (Section 2) and then discuss the model of definite description processing that we adopted as a result of that work and the general architecture of the system (Section 3). In Section 4 we discuss the heuristics that we developed for resolving anaphoric definite descriptions, recognizing discourse-new descriptions, and processing bridging descriptions, and, in Section 5, how the performance of these heuristics was evaluated using the annotated corpus. Finally, we present the final configuration of the two versions of the system that we developed (Section 6), review other systems that perform similar tasks (Section 7), and present our conclusions and indicate future work (Section 8).

\section{Preliminary Empirical Work}

As mentioned above, the architecture of our system is motivated by the results concerning definite description use in our corpus, discussed in Poesio and Vieira (1998). In this section we briefly review the results presented in that paper.

2 In fact, it is precisely because we are interested in identifying the types of commonsense reasoning actually used in language processing that we focused on definite descriptions rather than on other types of anaphoric expressions (such as pronouns and ellipsis) that can be processed much more effectively on the basis of syntactic information alone (Lappin and Leass 1994; Hardt 1997). 


\subsection{The Corpus}

We used a subset of the Penn Treebank I corpus (Marcus, Santorini, and Marcinkiewicz 1993) from the ACL/DCI CD-ROM, containing newspaper articles from the Wall Street Journal. We divided the corpus into two parts: one, containing about 1,000 definite descriptions, was used as a source during the development of the system; we will refer to these texts as Corpus $1 .^{3}$ The other part, containing about 400 definite descriptions, was kept aside during development and used for testing; we will refer to this subset as Corpus $2{ }^{4}$

\subsection{Classifications of Anaphoric Expressions}

The best-known studies of definite description use (Hawkins 1978; Prince 1992; Fraurud 1990; Löbner 1987; Clark 1977; Sidner 1979; Strand 1996) classify definite descriptions on the basis of their relation with their antecedent. A fundamental distinction made in these studies is between descriptions that denote the same discourse entity as their antecedent (which we will call anaphoric or, following Fraurud, subsequent mention), descriptions that denote an object that is in some way "associated" with the antecedent-for example, it is part of it, as in a car ... the wheel (these definite expressions are called "associative descriptions" by Hawkins and "inferrables" by Prince), and descriptions that introduce a new entity into the discourse.

In the case of semantic identity between definite description and antecedent, a further distinction can be made depending on the semantic relation between the predicate used in the description and that used for the antecedent. The predicate used in an anaphoric definite description may be a synonym of the predicate used for the antecedent (a house ... the home), a generalization/hypernym (an oak... the tree), and even, sometimes, a specialization/hyponym (a tree... the oak). In fact, the NP introducing the antecedent may not have a head noun at all, e.g., when a proper name is used, as in Bill Clinton... the president. We will use the term direct anaphora when both description and antecedent have the same head noun, as in a house... the house. Direct anaphors are the easiest definite descriptions for a shallow system to resolve; in all other cases, as well as when the antecedent and the definite description are related in a more indirect way, lexical knowledge, or more generally encyclopedic knowledge, is needed.

All of the classifications mentioned above also acknowledge the fact that not all definite descriptions depend on the previous discourse for their interpretation. Some refer to an entity in the physical environment, others to objects which are assumed to be known on the basis of common knowledge (Prince's "discourse-new/hearer-old" expressions, such as the pope), and still others are licensed by virtue of the semantics of their head noun and complement (as in the fact that Milan won the Italian football championship).

\subsection{A Study of Definite Description Use}

In the experiments discussed in Poesio and Vieira (1998) we asked our subjects to classify all definite description uses in our two corpora. These experiments had the dual objective of verifying how easy it was for human subjects to agree on the distinctions between definite descriptions just discussed, and producing data that we could use to evaluate the performance of a system. The classification schemes we used were simpler than those proposed in the literature just mentioned and were motivated, on

3 The texts in question are w0203, w0207, w0209, w0301, w0305, w0725, w0760, w0761, w0765, w0766, w0767, w0800, w0803, w0804, w0808, w0820, w1108, w1122, w1124, and w1137.

4 The articles in this second subset are w0766, wsj_0003, wsj_0013, wsj_0015, wsj_0018, wsj_0020, wsj_0021, wsj_0022, wsj_0024, wsj_0026, wsj_0029, wsj_0034, wsj_0037, and wsj_0039. 
the one hand, by the desire to make the annotation uncomplicated for the subjects employed in the empirical analysis and, on the other hand, by our intention to use the annotation to get an estimate of how well a system using only limited lexical and encyclopedic knowledge could do. ${ }^{5}$ We ran two experiments, using two slightly different classification schemes. In the first experiment we used the following three classes: ${ }^{6}$

- direct anaphora: subsequent-mention definite descriptions that refer to an antecedent with the same head noun as the description;

- bridging descriptions: definite descriptions that either (i) have an antecedent denoting the same discourse entity, but using a different head noun (as in house ... building), or (ii) are related by a relation other than identity to an entity already introduced in the discourse; ${ }^{7}$

- discourse-new: first-mention definite descriptions that denote objects not related by shared associative knowledge to entities already introduced in the discourse.

In the second experiment we treated all anaphoric definite descriptions as part of one class (direct anaphora + bridging (i)), and all inferrables as part of a different class (bridging (ii)), without significant changes in the agreement results.

Agreement among annotators was measured using the $K$ statistic (Siegel and Castellan 1988; Carletta 1996). $K$ measures agreement among $k$ annotators over and above chance agreement (Siegel and Castellan 1988). The $K$ coefficient of agreement is defined as:

$$
K=\frac{P(A)-P(E)}{1-P(E)}
$$

where $P(A)$ is the proportion of times the annotators agree, and $P(E)$ the proportion of times that we would expect them to agree by chance. The interpretation of $K$ figures is an open question, but in the field of content analysis, where reliability has long been an issue (Krippendorff 1980), $K>0.8$ is generally taken to indicate good reliability, whereas $0.68 \leq K<0.8$ allows tentative conclusions to be drawn. Carletta et al. (1997) observe, however, that in other areas, such as medical research, much lower levels of $K$ are considered acceptable (Landis and Koch 1977).

An interesting overall result of our study was that the most reliable distinction that our annotators could make was that between first-mention and subsequent-mention $(K=0.76)$; the measure of agreement for the three-way distinction just discussed was $K=0.73$. The second interesting result concerned the distribution of definite descriptions in the three classes above: we found that about half of the definite descriptions were discourse-new. The distribution of the definite descriptions in classes in our first experiment according to annotators $\mathrm{A}$ and $\mathrm{B}$ are shown in Tables 1 and 2, respectively. (Class IV includes cases of idiomatic expressions or doubts expressed by the annotators).

The third main result was that we found very little agreement between our subjects on identifying briding descriptions: in our second experiment, the agreement on

\footnotetext{
5 Previous attempts to annotate anaphoric relations had resulted in very low agreement levels; for example, in the coreference annotation experiments for MUC-6 (Sundheim 1995), relations other than identity were dropped due to difficulties in annotating them.

6 In this experiment, our subjects could also classify a definite description as "idiomatic" or "doubt" see tables below.

7 In Poesio and Vieira (1998), Hawkins's term "associative" was used for this class; but in fact, the definition we used for the class is closest to the sense of "bridging" used by Clark (1977).
} 
Table 1

Classification of definite descriptions according to Annotator A.

\begin{tabular}{lcc}
\hline Class & Total Number & Percentage of Total \\
\hline I. Direct anaphora & 294 & $28.27 \%$ \\
II. Bridging & 160 & $15.38 \%$ \\
III. Discourse new & 546 & $52 \%$ \\
IV. Others & 40 & $3.84 \%$ \\
Total & 1,040 & $100.00 \%$
\end{tabular}

Table 2

Classification of definite descriptions according to Annotator B.

\begin{tabular}{lcc}
\hline Class & Total Number & Percentage of Total \\
\hline I. Direct anaphora & 332 & $31.92 \%$ \\
II. Bridging & 150 & $14.42 \%$ \\
III. Discourse new & 549 & $52.78 \%$ \\
IV. Others & 9 & $0.86 \%$ \\
Total & 1,040 & $100.00 \%$
\end{tabular}

bridging descriptions was $K=0.24$. This was due in part to the fact that many definite descriptions could be classified in more than one class (e.g., either anaphoric or bridging, depending on which antecedent was chosen) and in part to the fact that in the case of descriptions indirectly related to their antecedents, the discourse might provide more than one distinct equally suitable anchor (Poesio and Vieira 1998). The most common classification problem is distinguishing between larger situation and bridging descriptions; see also Fraurud (1990) and Poesio and Vieira (1998).

\section{A Model of Definite Description Processing Inspired by Empirical Studies}

The results just discussed led us to adopt a model of definite descriptions processing advanced in Fraurud (1990) and further elaborated in Poesio and Vieira (1998), according to which interpreting definite descriptions in written discourse is not just a matter of checking whether there is a suitable antecedent for the description, but also involves a classification task: recognizing whether a description is, in Fraurud's terms, first-mention or subsequent-mention-or, in our terminology, direct anaphora, discourse-new, or bridging. The crucial aspect of Fraurud's proposal is the idea that interpreting definite descriptions is not just a matter of looking for an antecedent; separate rules for recognizing first-mention definite descriptions are needed as well.

The fact that there was so much disagreement about bridging descriptions and their anchors led us to try to keep the rules for processing them fairly separate from those for processing other types of descriptions and to attempt to use agreement measures to evaluate the performance of the system, in addition to more traditional precision and recall figures.

\subsection{Fraurud's Proposal and Our Model}

The results discussed above further support Fraurud's (1990) criticism of the approach to processing definite NPs based on the assumption that they are primarily anaphoric. Because of the large proportion of first-mention definites found in the texts she exam- 
ined, Fraurud $(1990,421)$ claims that:

a model where the processing of first-mention definites always involves a failing search for an already established discourse referent as a first step seems less attractive. A reverse ordering of the procedures is, quite obviously, no solution to this problem, but a simultaneous processing as proposed by Bosch and Geurts (1989) might be.

Fraurud proposes, contra Heim (1982), that processing a definite NP may involve establishing a new discourse entity. ${ }^{8}$ This new discourse entity may then be linked to one or more anchors in the text or to a background referent. ${ }^{9}$ Fraurud discusses the example of the description the king, interpreted relationally, encountered in a text in which no king has been previously mentioned. Lexicoencyclopedic knowledge would provide the information that a king is related to a period and a country; these would constitute the anchors. The selection of the anchors would identify the pertinent period and country, and this would make possible the identification of a referent: say, for the anchors 1989 and Sweden, the referent identified would be Carl Gustav XVI. ${ }^{10}$

The most interesting aspect of Fraurud's proposal is the hypothesis that firstmention definites are not necessarily recognized simply because no suitable antecedent has been found; independent strategies for recognizing them may be involved. This hypothesis is consistent with Löbner's proposal (Löbner 1987) that the fundamental property of a definite description is that it denotes a function (in a logical sense); this function can be part of the meaning assigned to the definite description by the grammar (as in the beginning of $X$ ), or can be specified by context (as in the case of anaphoric definites). Fraurud's and Löbner's ideas can be translated into a requirement that a system have separate methods or rules for recognizing discourse-new descriptions (and in particular, Löbner's "semantically functional" definites) in addition to rules for resolving anaphoric definite descriptions; these rules may run in parallel with the rules for resolving anaphoric definites, rather than after them.

Rather than deciding a priori on the question of whether the heuristic rules (in our case) for identifying discourse-new descriptions should be run in parallel with resolution or after it, we treated this as an empirical question. We made the architecture of the system fairly modular, so that we could both try different heuristics and try applying them in a different order, using the corpus for evaluation. We discuss all the heuristics that we tried in Section 4 , and our evaluation of them in Section 5 .

\subsection{Architecture of Our System}

The overall architecture of our system is shown in Figure 1. The system attempts to classify each definite description as either direct anaphora, discourse-new, or bridging description. In addition to this classification, the system tries to identify the antecedents of anaphoric descriptions and the anchors (Fraurud 1990) of bridging descriptions. The

8 Discourse entities are representations in the discourse model of entities explicitly mentioned (Webber 1979; Heim 1982).

9 Background referents are entities that have not been mentioned in the discourse-those entities that Grosz (1977) would call "elements of the implicit focus."

10 Fraurud does not explain what it is that justifies the use of definite descriptions, if not familiarity. In Poesio and Vieira (1998) we suggest that Löbner's proposal (Löbner 1987) seems to account for the most data. 


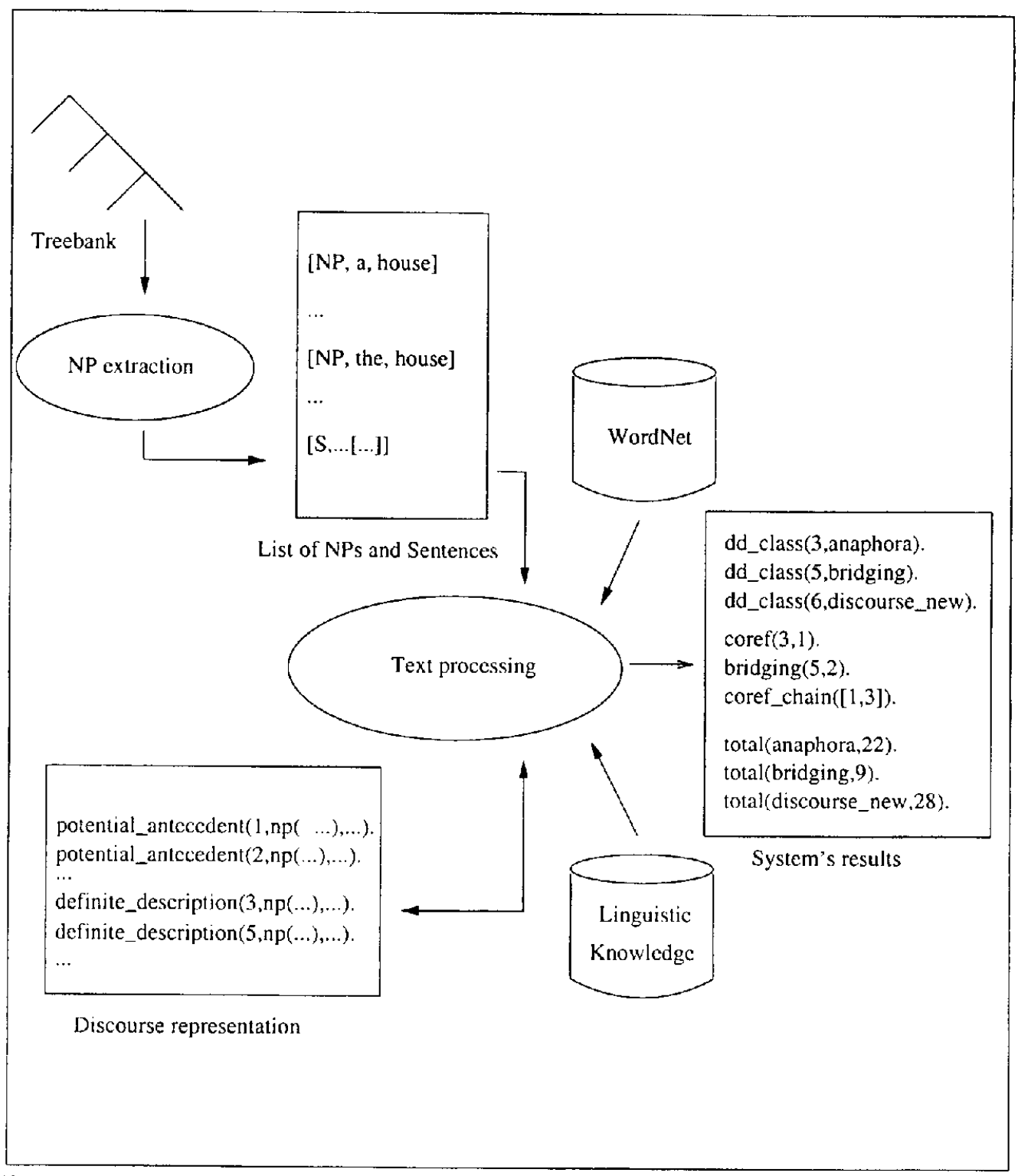

Figure 1

System architecture.

system processes parsed newswire texts from the Penn Treebank I, constructing a fairly simple discourse model that consists of a list of discourse entities that may serve as potential antecedents (which we call simply potential antecedents), according to the chosen segmentation algorithm (see below). The system uses the discourse model, syntactic information, and a small amount of lexical information to classify definite descriptions as discourse-new or to link them to anchors in the text; WordNet is also consulted by the version of the system that attempts to resolve bridging descriptions. The system is implemented in Sicstus Prolog. 
Input. The texts in the Penn Treebank corpus consist of parsed sentences represented as Lisp lists. During a preprocessing phase, a representation in Prolog list format is produced for each sentence, and the noun phrases it contains are extracted. The output of this preprocessing phase is passed to the system proper. For example, the sentence in (1) is represented in the Treebank as (2) and the input to the system after the preprocessing phase is (3). ${ }^{11}$ Note that all nested NPs are extracted, and that embedded NPs such as the Organization of Petroleum Exporting Countries are processed before the NPs that embed them (in this case, the squabbling within the Organization of Petroleum Exporting Countries).

(1) Mideast politics have calmed down and the squabbling within the Organization of Petroleum Exporting Countries seems under control for now.

(2) $\quad(\mathrm{s}(\mathrm{S}$

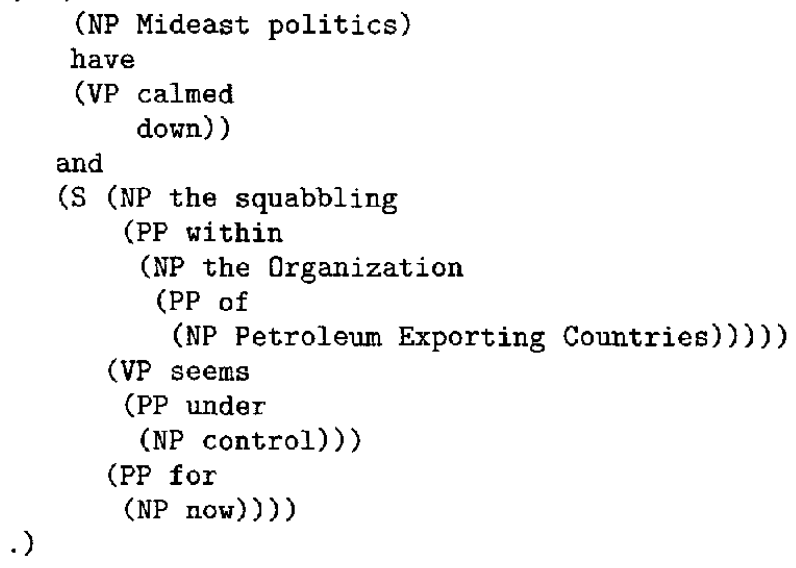

(3) [NP, Mideast, politics $]$.

[NP, Petroleum, Exporting, Countries].

[NP, the , Organization, [PP, of , [NP, Petroleum, Exporting, Countries]]] .

[NP, the , squabbling, [PP, wi thin, [NP, the, Organization, [PP, of , [NP, Petroleum, Exporting, Countries]]]]].

[NP, control].

[ [S, [S, [NP, Mideast, politics] , have, [VP, calmed, [PP, down] ] ] and, [S, [NP, the, squabbling, [PP, within, [NP, the , Organization, [PP, of , [NP, Petroleum, Exporting, Countries] ]] ] , [VP, seems, [PP, under, [NP, control]], $[P P$, for , now $]]],$.$] .$

Output. The system outputs the classification it has assigned to each definite description in the text, together with the coreferential and bridging links it has identified.

11 Prolog variables will be indicated in the rest of the paper by the use of "-" at the beginning and end of the variables; e.g., $X$ - for variable $X$. 


\section{The Heuristics}

We developed three types of heuristics:

- for directly resolving anaphoric descriptions. These include heuristics for dealing with segmentation and to handle modification.

- for identifying discourse-new descriptions. Some of these heuristics attempt to recognize semantically functional definite descriptions (Hawkins 1978; Löbner 1987), whereas others try to recognize definite descriptions that are anchored via their modification (Clark and Marshall 1981; Prince 1981).

- for identifying the anchor of a bridging description and the semantic relation between the bridging description and its anchor. WordNet is accessed, and heuristics for named entity recognition were also developed.

We present in turn the heuristics for each class of definite descriptions in this section, and discuss their limitations. The final configuration of the system was arrived at on the basis of an extensive evaluation of the heuristics using the corpus annotated in our previous work (Poesio and Vieira 1998). The evaluation was used both to determine which version of each heuristic worked better, and to identify the best order in which to try them.

\subsection{Direct Anaphora}

Our system's strategy for resolving direct anaphora is very simple: it just looks for a potential antecedent whose head matches the head noun of the definite description. The key issues to address in doing this are:

- how to identify the potential antecedents, and

- how to match the definite descriptions with the potential antecedents.

Performing each of these tasks may potentially involve complex syntactic analysis and general reasoning; we will discuss our heuristic solutions to them in turn.

4.1.1 Identifying Head Nouns. In order to resolve an anaphoric description, it is necessary to identify its head noun, which in the parsed texts of the Penn Treebank is generally the rightmost atom in the NP. For example, the nouns politics and squabbling are the heads of the following NPs:

(4)

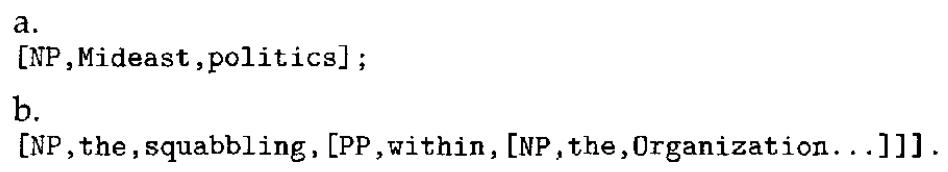

Although this strategy works most of the time, it does have some problems. One of these problems are headless definites (such as the highest in the southern region). A second problem are definites whose head is not represented in the Treebank as an atom at the determiner level (such as, [NP,The,[NP,[NP,2000],[NP,tax]]]). Corpus 1, for example, contains 17 definite descriptions with these problems $(0.2 \%)$. A third problem 
is coordination: for example, our algorithm does not recognize that a noun such as reporters below is a head noun:

[NP,reporters, and,editors,[PP,of,[NP,The,WS]]]].

4.1.2 Potential Antecedents. The second problem is to determine which NPs should be used to resolve definite descriptions, among all those in the text. The system keeps track of NP index, NP structure, head noun, and NP type (definite, indefinite, bare plural, possessive ${ }^{12}$ ) of each potential antecedent, as illustrated by (5) below.

$$
\text { potential_antecedent }(I, n p(N P), \operatorname{head}(H), \operatorname{type}(T)) \text {. }
$$

Examples of potential antecedents extracted from (6) are shown in (7):

(6) In an interview with reporters of the Wall Street Journal, the candidate appears quite confident of victory and of his ability to handle the mayoralty.

(7) a.

$$
\begin{aligned}
\text { potential_antecedent } & (1, \mathrm{np}(\text { NPstructure_), } \\
& \text { head(reporters), } \\
\text { type(indef)). } & \\
\text { potential_antecedent } & (2, \text { np(_NPstructure_), } \\
& \text { head(interview), } \\
& \text { type(indef)). }
\end{aligned}
$$

b.

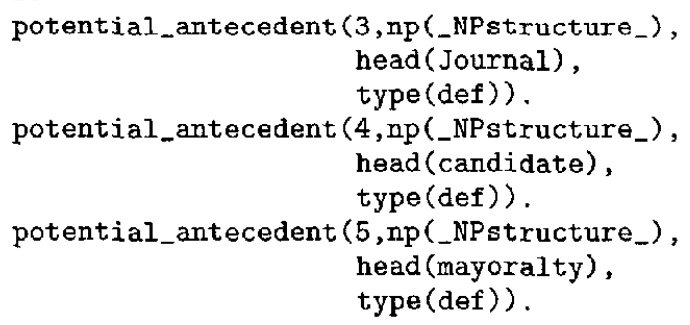

c.

potential_antecedent $(6, n p$ (_NPstructure_), head(ability), type(possessive)).

d. potential_antecedent
( 7, np (_NPstructure_),
head(victory),
type(other)).

We found that different recall/precision trade-offs can be achieved depending on the choice of potential antecedents-i.e., depending on whether all NPs are considered as possible antecedents, or only indefinite NPs, or various other subsets-so we ran experiments to identify the best group of potential antecedents. Four different NP

12 Other NPs not included in any of these categories are identified as type(other). 
subsets were considered:

1. indefinite NPs, defined as NPs containing the indefinite articles $a$, an, some and bare/cardinal plurals, as in (7a); ${ }^{13}$

2. indefinite NPs and definite descriptions (NPs beginning with the definite article) ((7a) and (7b));

3. indefinite NPs, definite descriptions, and possessive NPs (NPs with a possessive pronoun or possessive mark) ((7a), (7b) and (7c));

4. all NPs $((7 a),(7 b),(7 c))$ and $(7 d))$.

The results obtained by considering each subset of the total number of NPs as potential antecedents are discussed in Section 5.2.

4.1.3 Segmentation. The set of potential antecedents of anaphoric expressions is also restricted by the fact that antecedents tend to have a limited "life span"-i.e., they only serve as antecedents for anaphoric expressions within pragmatically determined segments of the whole text (see, for example, Reichman [1985], Grosz and Sidner [1986] and Fox [1987]). In our corpus we found that about $10 \%$ of direct anaphoric definite descriptions have more than one possible antecedent if segmentation is not taken into account (Vieira and Poesio 1996). In (8), for example, the antecedent of

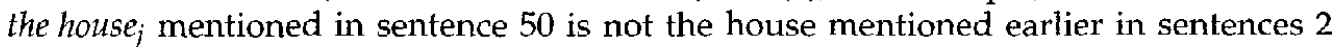
and 19, but another (nonmobile) house implicitly introduced in sentence 49 by the reference to the yard.

(8) 2. A deep trench now runs along its north wall, exposed when the house lurched two feet off its foundation during last week's earthquake.

19. Others grab books, records, photo albums, sofas and chairs, working frantically in the fear that an aftershock will jolt the house $e_{i}$ again.

20. The owners, William and Margie Hammack, are luckier than many others.

49. When Aetna adjuster Bill Schaeffer visited a retired couple in Oakland last Thursday, he found them living in a mobile home $e_{k}$ parked in front of their yard.

50. The house itself, located about 50 yards from the collapsed section of double-decker highway Interstate 880 , was pushed about four feet off its foundation and then collapsed into its basement.

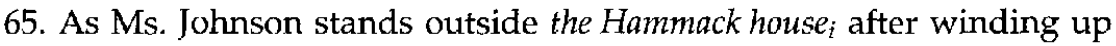
her chores there, the house $i$ begins to creak and sway.

13 Only plural nouns ending in s are handled by the system. 
In general, it is not sufficient to look at the most recent antecedents only: this is because segments are organized hierarchically, and the antecedents introduced in a segment at a lower level are typically not accessible from a segment at a higher level (Fox 1987; Grosz 1977; Grosz and Sidner 1986; Reichman 1985), whereas the antecedents introduced in a prior segment at the same level may be. Later in (8), for example, the house ${ }_{j}$ in sentence 50 becomes inaccessible again, and in sentence 65 , the text starts referring again to the house introduced in sentence 2. Automatically recognizing the hierarchical structure of texts is an unresolved problem, as it involves reasoning about intentions; ${ }^{14}$ better results have been achieved on the simpler task of "chunking" the text into sequences of segments, generally by means of lexical density measures (Hearst 1997; Richmond, Smith, and Amitay 1997).

The methods for limiting the life span of discourse entities that we considered for our system are even simpler. One type of heuristic we looked at are windowbased techniques, i.e., considering only the antecedents within fixed-size windows of previous sentences, although we allow some discourse entities to have a longer life span: we call this method loose segmentation. More specifically, a discourse entity is considered a potential antecedent for a definite description when the antecedent's head is identical to the description's head, and

- the potential antecedent is within the established window, or else

- the potential antecedent is itself a subsequent mention, or else

- the definite description and the antecedent are identical NPs (including the article).

We also considered an even simpler recency heuristic: this involves keeping a table indexed by the heads of potential antecedents, such that the entry for noun $\mathrm{N}$ contains the index of the last occurrence of an antecedent with head N. Finally, we considered combinations of segmentation and recency. (The results of these two heuristics are compared in Section 5.2.

4.1.4 Noun Modifiers. Once the head nouns of the antecedent and of the description have been identified, the system attempts to match them. This head-matching strategy works correctly in simple cases like (9):

(9) Grace Energy hauled a rig here ... The rig was built around 1980.

In general, however, when matching a definite description with a potential antecedent the information provided by the prenominal and the postnominal part of the noun phrases also has to be taken into account. For example, a blue car cannot serve as the antecedent for the red car, or the house on the left for the house on the right. In our corpus, cases of antecedents that would incorrectly match by simply matching heads without regarding premodification include:

\section{a. the business community ... the younger, more activist black political community;}

b. the population ... the voting population. 
Again, taking proper account of the semantic contribution of these premodifiers would, in general, require commonsense reasoning. For the moment, we only developed heuristic solutions to the problem, including:

- allowing an antecedent to match with a definite description if the premodifiers of the description are a subset of the premodifiers of the antecedent. This heuristic deals with definites that contain less information than the antecedent, such as an old Victorian house ... the house, and prevents matches such as the business community ... the younger, more activist black political community.

- allowing a nonpremodified antecedent to match with any same head definite. This second heuristic deals with definites that contain additional information, such as a check ... the lost check.

The information that two discourse entities are disjoint may come from postmodification, as well, although same head antecedents with different postmodification are not as common as those with differences in premodification. An example from our corpus is shown in (11).

$$
\begin{aligned}
& \text { a chance to accomplish several objectives ... the chance } \\
& \text { to demonstrate an entrepreneur } \\
& \text { like himself could run Pinkerton's better than an unfocused conglomerate or } \\
& \text { investment banker. }
\end{aligned}
$$

The heuristic method we developed to deal with postmodification is to compare the description and antecedent, preventing resolution in those cases where both are postmodified and the modifications are not the same. (These results are also discussed in Section 5.2.)

\subsection{Discourse-New Descriptions}

As mentioned above, a fundamental characteristic of our system is that it also includes heuristics for recognizing discourse-new descriptions (i.e., definite descriptions that introduce new discourse entities) on the basis of syntactic and lexical features of the noun phrase. Our heuristics are based on the discussion in Hawkins (1978), who identified a number of correlations between certain types of syntactic structure and discourse-new descriptions, particularly those he called "unfamiliar" definites (i.e., those whose existence cannot be expected to be known on the basis of generally shared knowledge), including: ${ }^{15}$

- the presence of "special predicates":

- the occurrence of premodifiers such as first or best when accompanied with full relatives, e.g., the first person to sail to America (Hawkins calls these "unexplanatory modifiers"; Löbner

15 Hawkins himself proposes a transformation-based account of unfamiliar definites, but the correlations he identified proved to be a useful source of heuristics for identifying these uses of definite descriptions even though the existence of counterexamples to these heuristics suggests that a syntactic-based account cannot be entirely correct. Most of these examples can be accounted for in terms of Löbner's theory of definiteness. 
[1987] showed how these predicates may license the use of definite descriptions in an account of definite descriptions based on functionality);

- a head noun taking a complement such as the fact that there is life on Earth (Hawkins calls this subclass "NP complements");

- the presence of restrictive modification, as in the inequities of the current land-ownership system.

Our system attempts to recognize these syntactic patterns. We also added heuristics classifying as unfamiliar some definites occurring in

- appositive constructions (e.g., Glenn Cox, the president of Phillips Petroleum Co.);

- copular constructions (e.g., the man most likely to gain custody of all this is a career politician named David Dinkins).

(The reason definite descriptions in appositive and copular constructions tend to be discourse-new, in fact unfamiliar, is that the information needed for the identification is given by the NP to which the apposition is attached and the predicative part of the copular construction, respectively. ${ }^{16}$ )

Finally, we found that three classes of what Hawkins called "larger situation" definites (those whose existence can be assumed to be known on the basis of encyclopedic knowledge, such as the pope) can also be recognized on the basis of heuristics exploiting syntactic and lexical features:

- definites that behave like proper nouns, like the United States;

- definites that have proper nouns in their premodification, such as the Iran-Iraq war;

- definites referring to time, such as the time or the morning.

In our corpus study we found that our subjects did much better at identifying discourse-new descriptions all together $(K=0.68)$ than they did at distinguishing unfamiliar from larger situation cases $(K=0.63)$. This finding was confirmed by our implementation: although each of the heuristics is designed, in principle, to identify only one of the uses (larger situation or unfamiliar), they work better at identifying together the whole class of discourse-new descriptions.

4.2.1 Special Predicates. Some cases of discourse-new definite descriptions can be identified by comparing the head noun or modifiers of the definite NP with a list of predicates that are either functional or likely to take a complement (Löbner 1987). Our list of predicates that, when taking NP complements, are generally used to introduce discourse-new entities, was compiled by hand and currently includes the nouns fact, result, conclusion, idea, belief, saying, and remark. In these cases, what licenses the use of a definite is not anaphoricity, but the fact that the head noun can be interpreted as

16 In the systems participating in MUC, definite descriptions occurring in appositions are treated as anaphoric on the preceding NP; our system considers the NP and the apposition as a unit that introduces a new referent to the discourse. 
semantically functional; the noun complement specifies the argument of the function. Functionality is enough to license the use of the definite description (Löbner 1987). An example of definite description classified as discourse-new on these grounds is given in (12).

Mr. Dinkins also has failed to allay Jewish voters' fears about his association with the Rev. Jesse Jackson, despite the fact that few local non-Jewish politicians have been as vocal for Jewish causes in the past 20 years as Mr. Dinkins has.

When encountering a definite whose head noun occurs in this list, the system checks if a complement is present or if the definite appears in a copular construction (e.g., the fact is that...).

A second list of special predicates consulted by the system includes what Hawkins called unexplanatory modifiers: these include adjectives such as first, last, best, most, maximum, minimum, and only and superlatives in general. ${ }^{17}$. All of these adjectives are predicate modifiers that turn a head noun into a function, therefore again-according to Löbner-licensing the use of a definite even when no antecedent is present (see examples below). When applying this heuristic, the system verifies the presence of a complement for some of the modifiers (first, last), but not for superlatives.
a. Mr. Ramirez just got the first raise he can remember in eight years, to $\$ 8.50$ an hour from $\$ 8$.
b. She jumps at the slightest noise.

Finally, our system uses a list of special predicates that we found to correlate well with larger situation uses (i.e., definite descriptions referring to objects whose existence is generally known). This list consists mainly of terms indicating time reference, and includes the nouns hour, time, morning, afternoon, night, day, week, month, period, quarter, year, and their respective plurals. An example from the corpus is:

(14) Only 14,505 wells were drilled for oil and natural gas in the U.S. in the first nine months of the year.

Other definites typically used with a larger situation interpretation are the moon, the sky, the pope, and the weather.

It should be noted that although these constructions may indicate a discoursenew interpretation, these expressions may also be used anaphorically; this is one of the cases in which a decision has to be made concerning the relative priority of different heuristics. We discuss this issue further in connection with the evaluation of the system's performance in Section $5 .^{18}$

4.2.2 Restrictive and Nonrestrictive Modification. A second set of heuristics for identifying discourse-new descriptions that we derived from Hawkins's suggestions and

17 The list should be made more comprehensive; so far it includes the cases observed in the corpus analysis and a few other similar modifiers.

18 Morc recently, Bean and Riloff (1999) have proposed methods for automatically extracting from a corpus heads that correlate well with discourse novelty. 
Table 3

Distribution of prepositional phrases and relative clauses.

\begin{tabular}{lrc}
\hline Restrictive Postmodification & $\#$ & $\%$ \\
\hline Prepositional phrases & 152 & $77 \%$ \\
Relative clauses & 45 & $23 \%$ \\
Total & 197 & $100 \%$
\end{tabular}

from our corpus analysis look for restrictive modification..$^{19}$ We developed patterns to recognize restrictive postmodification and nonrestrictive postmodification; we also tested the correlation between discourse novelty and premodification. We discuss each of these heuristics in turn.

Restrictive Postmodification. Hawkins (1978) pointed out that unfamiliar definites often include referent-establishing relative clauses and associative clauses, while warning that not all relative clauses are referent-establishing. Some statistics about this correlation were reported by Fraurud (1990): she found that in her corpus $75 \%$ of complex definite NPs (i.e., modified by genitives, postposed PPs, restrictive adjectival modifiers) were first-mention. A great number of definite descriptions with restrictive postmodifiers are unfamiliar in our corpus as well (Poesio and Vieira 1998); in fact, restrictive postmodification was found to be the single most frequent feature of first-mention descriptions. Constructions of this type are good indicators of discourse novelty because a restrictive postmodifier may license the use of a definite description either by providing a link to the rest of the discourse (as in Prince's "containing inferrables") or by making the description into a functional concept. Looking for restrictive postmodifiers might therefore be a good way of identifying discourse-new descriptions.

The distribution of restrictive postmodifiers in our corpus is shown in Table 3; examples of each type of postmodifier are given below.

Relative clauses: these are finite clauses sometimes (but not always) introduced by relative pronouns such as who, whom, which, where, when, why, and that:
a. The place where he lives...
b. The guy we met ...

Nonfinite postmodifiers: these include ing, ed (participle), and infinitival clauses.
a. The man writing the letter is my friend.
b. The man to consult is Wilson.

Prepositional phrases and of-clauses: Quirk et al. (1985) found that prepositional phrases are the most common type of postmodification in English - three or four times more frequent than either finite or nonfinite clausal postmodification. This was confirmed by our corpus study (see

19 The term restrictive modification is used when the modifier provides information that is essential to identify the discourse entity referred to by the NP (Quirk et al. 1985). The modification is nonrestrictive when the head provides sufficient information to identify the discourse entity, so that the information provided by the modification is not essential for identification. 


\section{Table 4}

Distribution of prepositions (1).

\begin{tabular}{lrr}
\hline Prepositional Phrases & \multicolumn{1}{l}{$\#$} & \multicolumn{1}{c}{$\%$} \\
\hline Of-phrases & 120 & $79 \%$ \\
Other prepositions & 32 & $21 \%$ \\
Total & 152 & $100 \%$
\end{tabular}

Table 3). The types of prepositions observed for 188 postmodified descriptions are shown in Table 4; of-clauses are the most common.

Our program uses the following patterns to identify restrictive postmodifiers: ${ }^{20}$

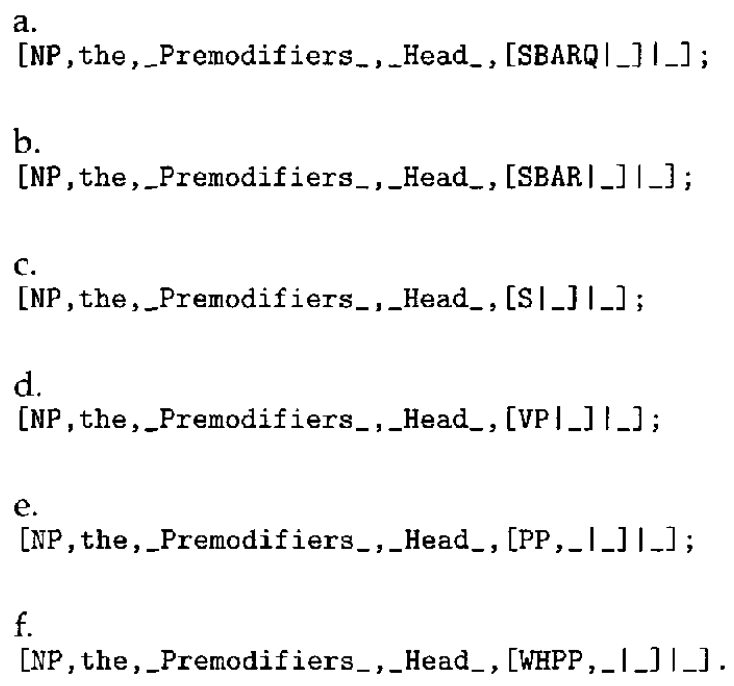

In the Treebank, sometimes the modified NP is embedded in another NP, so structures like (18) are also considered (again for all types of clauses just shown above):

$$
\left[N P,\left[N P, \text { the,_Premodifiers }{ }_{-}, H_{\text {Head_ }}\right],[\text { Clause }]\right. \text {. }
$$

Nonrestrictive postmodification. We found it important to distinguish restrictive from nonrestrictive postmodification, since in our corpus, definite descriptions with nonrestrictive postmodifiers were generally not discourse-new. Our system recognizes nonrestrictive postmodifiers by the simple yet effective heuristic of looking for commas. This heuristic correctly recognizes nonrestrictive postmodification in cases like:

(19) The substance, discovered almost by accident, is very important.

which are annotated in the Penn Treebank I as follows:

$$
\begin{aligned}
& \text { [NP, the , proposal , ' , ' [SBAR, [WHNP, which] , also, [S, [NP, T] , would, } \\
& \text { [VP, create, [NP, a, new , type, [PP, of , [NP, individual, } \\
& \text { retirement, account]]]]] ], ', ' ]... }
\end{aligned}
$$

20 Note that an NP may have zero, one, or more premodifiers. 
Restrictive Premodification. Restrictive modification is not as common in prenominal position as in posthead position, but it is often used, and was also found to correlate well with larger situation and unfamiliar uses of definite descriptions (Poesio and Vieira 1998). A restrictive premodifier may be a noun (as in (21)), a proper noun, or an adjective. ${ }^{21}$ Sometimes numerical figures (usually referring to dates) are used as restrictive premodifiers, as in (22)).

(21) A native of the area, he is back now after riding the oil-field boom to the top, then surviving the bust running an Oklahoma City convenience store. the 1987 stock market crash;

The heuristic we tested was to classify definite descriptions premodified by a proper noun as larger situation.

4.2.3 Appositions. During our corpus analysis we found additional syntactic patterns that appeared to correlate well with discourse novelty yet had not been discussed by Hawkins, such as definite descriptions occurring in appositive constructions: they usually refer to the NP modified by the apposition, therefore there is no need for the system to look for an antecedent. Appositive constructions are treated in the Treebank as NP modifiers; therefore the system recognizes an apposition by checking whether the definite occurs in a complex noun phrase with a structure consisting of a sequence of noun phrases (which might be separated by commas, or not) one of which is a name or is premodified by a name, as in the examples in (23).
a. Glenn Cox, the president of Phillips Petroleum
b. [NP, [NP, Glenn, Cox ] , ' ', [NP, the, president, [PP, of , [NP , Phillips, Petroleum] ]] ;
c. the oboist, Heinz Holliger
d. [NP, [NP, the, oboist] , [NP, Heinz, Holliger] ] .

In fact a definite description may itself be modified by an apposition, e.g., an indefinite NP, as shown by (24). Such cases of appositive constructions are also recognized by the system.

the Sandhills Luncheon Cafe, a tin building in midtown.

Other examples of apposition recognized by the system are:
a. the very countercultural chamber group Tashi;
b. the new chancellor, John Major;
c. the Sharpshooter, a freshly drilled oil well two miles deep;

21 Our system cannot distinguish adjectives or verbs from nouns in premodification because it works directly off the parsed version of the Treebark, without looking at part-of-speech tags. 
4.2.4 Copular Phrases. Copular phrases such as the Prime Minister is Tony Blair also often involve discourse-new descriptions. We developed the following heuristic for handling copula constructions. If a description occurs in subject position, the system looks at the VP. If the head of the VP is the verb to be, to seem, or to become, and the complement of the verb is not an adjectival phrase, the system classifies the description as discourse-new. Two examples correctly handled by this heuristic are shown in (26); the syntactic representation of these cases in the Penn Treebank I is shown in (27).

a. The bottom line is that he is a very genuine and decent guy.

b. When the dust and dirt settle in an extra-nasty mayoral race, the man most likely to gain custody of all this is a career politician named David Dinkins.

$[S,[N P$, The , bottom, line $],[\mathrm{VP}$, is , [NP , [SBAR, that ... ] ] ] ] $]$.

If the complement of the verb is an adjective, the subject is typically interpreted referentially and should not be considered discourse-new on the basis of its complement (e.g., The president of the US is tall). Adjectival complements are represented as follows in the Treebank:

$$
[\mathrm{S},[\mathrm{NP}, \text { The , missing, watch }],[\mathrm{VP}, \text { is , [ADJP, emblematic...] ]] . }
$$

Definite descriptions in object position of the verb to be, such as the one shown in (29), are also considered discourse-new.

What the investors object to most is the effect they say the proposal would have on their ability to spot telltale "clusters" of trading activity.

4.2.5 Proper Names. Proper names preceded by the definite article, such as (30), are common in the genre we are dealing with, newspaper articles.

\section{the Securities and Exchange Commission.}

The first appearance of these definite descriptions in the text is usually a discoursenew description; subsequent mentions of proper names are regarded as cases of anaphora. To recognize proper names, the system simply checks whether the head is capitalized. If the test succeeds, the definite is classified as a larger situation use. ${ }^{22}$

\subsection{Bridging Descriptions}

Bridging descriptions are the definite descriptions that a shallow processing system is least equipped to handle. Linguistic and computational theories of bridging descriptions identify two main subtasks involved in their resolution: finding the element in the text to which the bridging description is related (anchor) and identifying the relation (link) holding between the bridging description and its anchor (Clark 1977; Sidner 1979; Heim 1982; Carter 1987; Fraurud 1990; Strand 1996). The speaker is licensed to use a bridging description when he or she can assume that the commonsense

22 Note that this test is performed just after trying to find an antecedent, so that the second instance of the same proper (head) noun will be classified as an anaphoric use. 
knowledge required to identify the relation is shared by the listener (Hawkins 1978; Clark and Marshall 1981; Prince 1981). This dependence on commonsense knowledge means that, in general, a system can only resolve bridging descriptions when supplied with an adequate knowledge base; for this reason, the typical way of implementing a system for resolving bridging descriptions has been to restrict the domain and feed the system with hand-coded world knowledge (see, for example, Sidner [1979] and especially Carter [1987]). A broader view of bridging phenomena (not only bridging descriptions) is presented in Hahn, Strube, and Markert (1996). They make use of a knowledge base from which they extract conceptual links to feed an adaptation of the centering model (Grosz, Joshi, and Weinstein 1995).

The relation between bridging descriptions and their anchors may be arbitrarily complex (Clark 1977; Sidner 1979; Prince 1981; Strand 1996), and the same description may relate to different anchors in a text: this makes it difficult to decide what the intended anchor and the intended link are (Poesio and Vieira 1998). For all these reasons, this class has been the most challenging problem we have dealt with in the development of our system, and the results we have obtained so far can only be considered very preliminary. Nevertheless, we feel that trying to process these definite descriptions is the only way to discover which types of commonsense knowledge are actually needed.

\subsection{Types of Bridging Descriptions}

Our work on bridging descriptions began with the development of a classification of bridging descriptions (Vieira and Teufel 1997) according to the kind of information needed to resolve them, rather than on the basis of the possible relations between descriptions and their anchors as is typical in the literature. This allowed us to get an estimate of what types of bridging descriptions we might expect our system to resolve. The classification is as follows:

- cases based on well-defined lexical relations, such as synonymy, hypernymy, and meronymy, that can be found in a lexical database such as WordNet (Fellbaum 1998), as in the flat ... the living room;

- bridging descriptions in which the antecedent is a proper name and the description a common noun, whose resolution requires some way of recognizing the type of object denoted by the proper name, as in Bach ... the composer;

- cases in which the anchor is not the head noun but a noun modifying an antecedent, as in the company has been selling discount packages ... the discounts

- cases in which the antecedent (anchor) is not introduced by an NP but by a VP, as in Kadane oil is currently drilling two oil wells. The activity...

- descriptions whose antecedent is not explicitly mentioned in the text, but is implicitly available because it is a discourse topic, e.g., the industry in a text referring to oil companies;

- cases in which the relation with the anchor is based on more general commonsense knowledge, e.g., about cause-consequence relations.

In the rest of this section, we describe the heuristics we developed for handling the first three of these classes: lexical bridges, bridges based on names, and bridges 
to entities introduced by nonhead nouns in a compound nominal (Poesio, Vieira, and Teufel 1997).

4.4.1 Bridging Descriptions and WordNet. In order to get a system that could be evaluated on a corpus containing texts in different domains, we used WordNet (Fellbaum 1998) as an approximation of a lexical knowledge source. We developed a WordNet interface (Vieira and Teufel 1997) that reports a possible semantic link between two nouns when one of the following is true:

- the nouns are in the same synset (i.e., they are synonyms of each other), as in suit/lawsuit;

- the nouns are in a hyponymy/hypernymy relation with each other, as in dollar/currency;

- there is a direct or indirect meronymy/holonymy (part of/has parts) relation between them, as in door/house;

- the nouns are coordinate sisters, i.e. hyponyms of the same hypernym, such as home/house, which are hyponyms of housing, lodging.

Sometimes, finding a relation between two predicates involves complex searches through WordNet's hierarchy. For example, there may be no relation between two head nouns, but there is a relation between compound nouns in which these nouns appear: thus, there is no semantic relation between record/album, but only a synonymy relation between record album/album. We found that extended searches of this type, or searches for indirect meronymy relations, yielded extremely low recall and precision at a very high computational cost; both types of search were dropped at the beginning of the tests we ran to process the corpus consulting WordNet (Poesio, Vieira, and Teufel 1997). The results of our tests with WordNet are presented in Section 5.4.

4.4.2 Bridging Descriptions and Named Entity Recognition. Definite descriptions that refer back to entities introduced by proper names (such as Pinkerton Inc ... the company) are very common in newspaper articles. Processing such descriptions requires determining an entity type for each name in the text, that is, if we recognize Pinkerton Inc. as an entity of type company, we can then resolve the subsequent description the company, or even a description such as the firm by finding a synonymy relation between company and firm using WordNet.

This so-called named entity recognition task has received considerable attention recently (Mani and MacMillan 1996; McDonald 1996; Paik et al. 1996; Bikel et al. 1997; Palmer and Day 1997; Wacholder and Ravin 1997; Mikheev, Moens, and Grover 1999) and was one of the tasks evaluated in the Sixth and Seventh Message Understanding Conferences. In MUC-6, 15 different systems participated in the competition (Sundheim 1995). For the version of the system discussed and evaluated here, we implemented a preliminary algorithm for named entity recognition that we developed ourselves; a more recent version of the system (Ishikawa 1998) uses the named entity recognition software developed by HCRC for the MUC-7 competition (Mikheev, Moens, and Grover 1999).

WordNet contains the types of a few names-typically, of famous people, countries, states, cities, and languages. Other entity types can be identified using appositive constructions and abbreviations (such as $\mathrm{Mr}$., Co., and Inc.) as cues. Our algorithm for assigning a type to proper names is based on a mixture of the heuristics just described. 
The system first looks for the above-mentioned cues to try to identify the name type. If no cue is found, pairs consisting of the proper name and each of the elements from the list country, city, state, continent, language, person are consulted in our WordNet interface to verify the existence of a semantic relation.

The recall of this algorithm was increased by including a backtracking mechanism that reprocesses a text, filling in the discourse representation with missing name types. With this mechanism we can identify later the type for the name Morishita in a textual sequence in which the first occurrence of the name does not provide surface indication of the entity type: e.g., Morishita ... Mr. Morishita. The second mention includes such a clue $(M r$.); by processing the text twice, we recover such missing types.

After finding the types for names, the system uses the techniques previously described for same-head matching or WordNet lookup to match the descriptions with the types found for previous named entities.

4.4.3 Compound Nouns. Sometimes, the anchor for a bridging description is a nonhead noun in a compound noun: stock market crash ... the markets;

One way to process these definite descriptions would be to update the discourse model with discourse referents not only for the NP as a whole, but also for the embedded nouns. For example, after processing stock market crash, we could introduce a discourse referent for stock market, and another discourse referent for stock market crash. ${ }^{23}$ The description the markets would be coreferring with the first of these referents (with an identical head noun), and then we could simply use our anaphora resolution algorithms. This solution, however, makes available discourse referents that are generally inaccessible for anaphora (Postal 1969). For example, it is generally accepted that in (32), a deer is not accessible for anaphoric reference. ${ }^{24}$

I saw [a deer, hunter $]_{j} . I t_{i}^{*}$ was dead.

Therefore, we followed a different route. Our algorithm for identifying anchors attempts to match not only heads with heads, but also:

1. The head of a description with the premodifiers of a previous NP:

$$
\text { the stock market crash ... the markets; }
$$

2. The premodifiers of a description with the premodifiers of its antecedents:

$$
\text { his art business... the art gallery. }
$$

3. And finally, the premodifiers of the description with the head of a previous NP:

$$
\text { a 15-acre plot and main home... the home site. }
$$

23 Note that the collection of potential antecedents containing all NPs will just have the NP head crash for stock market crash. The system considers the whole NP structure as only one discourse referent, according to the structure of the Penn Treebank: [NP,the,1987,stock,market,crash].

24 These proposed constraints have been challenged by Ward, Sproat, and McKoon (1991). 


\section{Evaluation of the Heuristics}

In this section we discuss the tests we ran to arrive at a final configuration of the system. The performance of the heuristics discussed in Section 4 was evaluated by comparing the results of the system with the human annotation of the corpus produced during the experiments discussed in Poesio and Vieira (1998). Several variants of our heuristics were tried using Corpus 1 as training data; after deciding upon an optimal version, our algorithms were evaluated using Corpus 2 as test data. Because our proposals concerning bridging descriptions are much less developed than those concerning anaphoric descriptions and discourse-new descriptions, we ran separate evaluations of two versions of the system: Version 1, which does not attempt to resolve bridging descriptions, and Version 2, which does; we will point out below which version of the system is considered in each evaluation.

\subsection{Evaluation Methods}

The fact that the annotators working on our corpus did not always agree either on the classification of a definite description or on its anchor raises the question of how to evaluate the performance of our system. We tried two different approaches: evaluating the performance of the system by measuring its precision and recall against a standardized annotation based on majority voting (as done in MUC), and measuring the extent of the system's agreement with the rest of the annotators by means of the same metric used to measure agreement among the annotators themselves (the kappa statistic). We used the first form of evaluation to measure both the performance of the single heuristics and the performance of the system as a whole; the agreement measure was only used to measure the overall performance of the system. We discuss each of these in turn. ${ }^{25}$

5.1.1 Precision and Recall. Recall and precision are measures commonly used in Information Retrieval to evaluate a system's performance. Recall is the percentage of correct answers reported by the system in relation to the number of cases indicated by the annotated corpus:

$$
R=\frac{\text { number of correct responses }}{\text { number of cases }}
$$

whereas precision is the percentage of correctly reported results in relation to the total reported:

$$
P=\frac{\text { number of correct responses }}{\text { number of responses }}
$$

These two measures may be combined to form one measure of performance, the $F$ measure, which is computed as follows:

$$
F=\frac{(W+1) R P}{(W R)+P}
$$

$W$ represents the relative weight of recall to precision and typically has the value 1 . A single measure gives us a balance between the two results; $100 \%$ of recall may be due to a precision of $0 \%$ and vice versa. The $F$ measure penalizes both very low recall and very low precision.

25 For a rather thorough discussion of the problem of evaluating anaphora resolution algorithms, see Mitkov (2000). 
5.1.2 Semiautomatic Evaluation against a Standardized Annotation. The precision and recall figures for the different variants of the system were obtained by comparing the classification produced by each version with a standardized annotation, extracted from the annotations produced by our human annotators by majority judgement: whenever at least two of the three coders agreed on a class, that class was chosen. Details of how the standard annotation was obtained are given in Vieira (1998) ${ }^{26}$

The system's performance as a classifier was automatically evaluated against the standard annotation of the corpus as follows. Each NP in a text is given an index:

$$
\text { A house }^{106} \ldots \text { The house }{ }^{135} \ldots
$$

When a text is annotated or processed, the coder or system associates each index of a definite description with a type of use; both the standard annotation and the system's output are represented as Prolog assertions.

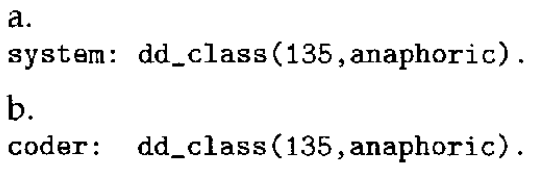

To assess the system's performance on the identification of a coreferential antecedent, it is necessary to compare the links that indicate the antecedent of each description classified as anaphora. These links are also represented as Prolog assertions, as follows:

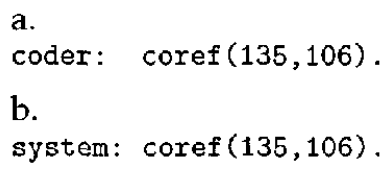

The system uses these assertions to build an equivalence class of discourse entities, called a coreference chain. When comparing an antecedent indicated by the system for a given definite description with that in the annotated corpus, the corresponding coreference chain is checked-that is, the system's indexes and the annotated indexes do not need to be exactly the same as long as they belong to the same coreference chain. In this way, both (40a) and (40b) would be evaluated as correct answers if the corpus is annotated with the links shown in (39).

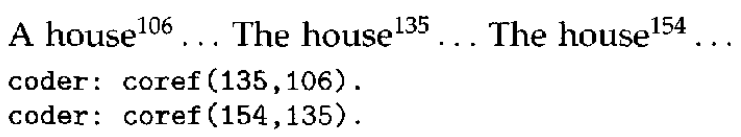

a.

system: coref $(154,135)$.

b.

system: coref $(154,106)$.

26 An alternative method is to give fractional values to a classification depending on the number of agreements (Hatzivassiloglou and McKeown (1993). 
In the end, we still need to check the results manually, because our annotated coreference chains are not complete: our annotators did not annotate all types of anaphoric expressions, so it may happen that the system indicates as antecedent an element outside an annotated coreference chain, such as a bare noun or possessive. In (41), for example, suppose that all references to the house are coreferential:

$$
\begin{aligned}
& \text { A house }^{106} \ldots \text { The house } \\
& \text { coref }(154,140) \text {. }
\end{aligned}
$$

If NP 135 is indicated as the antecedent for NP 154 in the corpus annotation (so that 140 is not part of the annotated coreference chain), and the system indicates 140 as the antecedent for 154, an error is reported by the automatic evaluation, even though all of these NPs refer to the same entity. A second consequence of the fact that the coreference chains in our standard annotation are not complete is that in the evaluation of direct anaphora resolution, we only verify if the antecedents indicated are correct; we do not evaluate how complete the coreferential chains produced by the system are. By contrast, in the evaluation of the MUC coreference task, where all types of referring expressions are considered, the resulting co-reference chains are evaluated, rather than just the indicated antecedent (Vilain et al. 1995). Even our limited notion of coreference chain was, nevertheless, very helpful in the automatic evaluation, considerably reducing the number of cases to be checked manually.

5.1.3 Measuring the Agreement of the System with the Annotators. Because the agreement between our annotators in Poesio and Vieira (1998) was often only partial, in addition to precision and recall measures, we evaluated the system's performance by measuring its agreement with the annotators using the $K$ statistic we used in Poesio and Vieira (1998) to measure agreement among annotators. Because the proper interpretation of $K$ figures is still open to debate, we interpret the $K$ figures resulting from our tests comparatively, rather than absolutely, (by comparing better and worse levels of agreement).

\subsection{Anaphora Resolution}

We now come to the results of the evaluation of alternative versions of the heuristics dealing with the resolution of direct anaphora (segmentation, selection of potential antecedents, and premodification) discussed in Section 4.1. The optimal version of our system is based on the best results we could get for resolving direct anaphora, because we wanted to establish the coreferential relations among discourse NPs as precisely as possible.

5.2.1 Life Span of Discourse Entities. In Section 4.1 we discussed two heuristics for limiting the life span of discourse entities. The first segmentation heuristic discussed there, loose segmentation, is window based, but the restriction on sentence distance is relaxed (i.e., the resolver will consider an antecedent outside the window) when either:

- the antecedent is itself a subsequent-mention; or

- the antecedent is identical to the definite description being resolved (including the article).

With loose segmentation, it is possible for the system to identify more than one coreference link for a definite description: all antecedents satisfying the requirements 
Table 5

Evaluation of loose segmentation and recency heuristics.

\begin{tabular}{llll}
\hline Heuristics & $\mathbf{R}$ & $\mathbf{P}$ & $\mathbf{F}$ \\
\hline Segmentation: 1-sentence window & $71.79 \%$ & $86.48 \%$ & $78.45 \%$ \\
Segmentation: 4-sentence window & $76.92 \%$ & $82.75 \%$ & $79.73 \%$ \\
Segmentation: 8-sentence window & $78.20 \%$ & $80.26 \%$ & $79.22 \%$ \\
Recency: all sentences & $80.76 \%$ & $78.50 \%$ & $79.62 \%$
\end{tabular}

Table 6

Evaluation of the strict segmentation heuristic.

\begin{tabular}{llll}
\hline Strict Segmentation & $\mathbf{R}$ & $\mathbf{P}$ & $\mathbf{F}$ \\
\hline 1-sentence window & $29.48 \%$ & $89.32 \%$ & $44.33 \%$ \\
4-sentence window & $57.69 \%$ & $88.23 \%$ & $69.76 \%$ \\
8-sentence window & $67.94 \%$ & $84.46 \%$ & $75.31 \%$
\end{tabular}

within the current window will be indicated as a possible antecedent. Therefore, when evaluating the system's results, we may find that all antecedents indicated for the resolution of a description were right, or some were right and some wrong, or that all were wrong. The recall and precision figures reported here relate to those cases were all resolutions indicated were right according to the annotated corpus.

In Section 4.1 we also discussed a second segmentation heuristic, which we called recency: the system does not collect all candidate NPs as potential antecedents, but only keeps the last occurrence of an NP from all those having the same head noun, and there are no restrictions regarding the antecedent's distance.

The results of these two methods for different window sizes are shown in Table 5 . The results in this table were obtained by considering as potential antecedents indefinites (i.e., NPs with determiners $a$, an, and some; bare NPs; and cardinal plurals), possessives, and definite descriptions, as in Vieira and Poesio (1996); we also used the premodification heuristics proposed there. Alternatives to these heuristics were also evaluated; the results are discussed later in this section.

The resulting $F$ measures were almost the same for all heuristics, but there was clearly an increase in recall with a loss of precision when enlarging the window size. ${ }^{27}$ The recency heuristic had the best recall, but the lowest precision, although not much lower than the others. The best precision was achieved with a one-sentence window, and recall was not dramatically affected, but this only happened because the window size constraint was relaxed.

To show what happens when a strict version of the window-based segmentation approach is used, consider Table 6. (Strict segmentation means that the system only considers those antecedents that are inside the sentence window for resolving a description, with no exceptions.) As the table shows, this form of segmentation results in higher precision, but has a strong negative effect on recall. The overall $F$ values are all worse than for the heuristics in Table 5.

Finally, we tried a combination of the recency and segmentation heuristics: just one potential antecedent for each different head noun is available for resolution, the last

27 In our experiments small differences in recall, precision, and $F$ measures are frequent. We generally assume in this paper that such differences are not significant, but a more formal significance test along the lines of that in Chinchor (1995) will eventually be necessary to verify this. 
Table 7

Combining loose segmentation and recency heuristics.

\begin{tabular}{llll}
\hline Combined Heuristics & $\mathbf{R}$ & $\mathbf{P}$ & $\mathbf{F}$ \\
\hline 4 sentences + recency & $75.96 \%$ & $87.77 \%$ & $81.44 \%$ \\
8 sentences + recency & $77.88 \%$ & $84.96 \%$ & $81.27 \%$
\end{tabular}

Table 8

Evaluation of the heuristics for choosing potential antecedents.

\begin{tabular}{llll}
\hline Antecedents Selection & $\mathbf{R}$ & $\mathbf{P}$ & $\mathbf{F}$ \\
\hline Indefinites, definite descriptions, and possessives & $75.96 \%$ & $87.77 \%$ & $81.44 \%$ \\
All NPs & $77.88 \%$ & $86.17 \%$ & $81.81 \%$ \\
Indefinites and definite descriptions & $73.39 \%$ & $88.41 \%$ & $80.21 \%$ \\
Indefinites only & $12.17 \%$ & $77.55 \%$ & $21.05 \%$
\end{tabular}

occurrence of that head noun. The resolution still respects the segmentation heuristic (loose version). The results are presented in Table 7. This table shows that by combining the recency and loose segmentation approaches to segmentation we obtain a better trade-off between recall and precision than using each heuristic separately. The version with higher $F$ value in Table 7 (four-sentence window plus recency) was chosen as standard and used in the tests discussed in the rest of this section.

5.2.2 Potential Antecedents. Next, we evaluated the various ways of restricting the set of potential antecedents discussed in Section 4.1, using four-sentence-window loose segmentation with recency. In an earlier version of the system (Vieira and Poesio 1996), only those definite descriptions that were not resolved with a same-head antecedent were considered as potential antecedents; resolved definite descriptions would be linked to previous NPs, but would not be made available for subsequent resolution. (The idea was that the same antecedent used in one resolution could be used to resolve all subsequent mentions cospecifying with that definite description.) An important difference between that implementation and the current one is that in the new version, the definites resolved by the system are also made available as potential antecedents of subsequent definites. This is because in our previous prototype, errors in identifying an indefinite antecedent were sometimes propagated through a coreference chain, so that the right antecedent would be missed. The results are shown in Table 8.

If we only consider indefinites as potential antecedents, recall is extremely low $(12 \%)$; we also get the worst precision. In other words, considering only indefinites for the resolution of definite descriptions is too restrictive; this is because our corpus contains a large number of first-mention definite descriptions that serve as antecedents for subsequent references (similar results were also reported in Fraurud [1990]). The version with the highest precision $(88 \%)$ is the one that only considers indefinites and definite descriptions as antecedents, but recall is lower compared to the version that considered other NPs. We chose, as the basis for further testing, a version that combines near-optimal values for $F$ and precision, i.e., the version that takes indefinites, definite descriptions, and possessives (first row in Table 8).

5.2.3 Premodifiers. Finally, we tested our heuristics for dealing with premodifiers. We tested the matching algorithm from Vieira and Poesio (1996) in the present version of the system; the results are presented in Table 9. In that table, we also show the 
Table 9

Evaluation of the heuristics for premodification (Version 1).

\begin{tabular}{llll}
\hline Antecedents Selection & $\mathbf{R}$ & $\mathbf{P}$ & $\mathbf{F}$ \\
\hline 1. Ant-set/Desc-subset & $69.87 \%$ & $91.21 \%$ & $79.12 \%$ \\
2. Ant-empty & $55.12 \%$ & $88.20 \%$ & $67.85 \%$ \\
3. Ant-subset/Desc-set & $64.74 \%$ & $88.59 \%$ & $74.81 \%$ \\
1 and 2 (basic v.) & $75.96 \%$ & $87.77 \%$ & $81.44 \%$ \\
1 and 3 & $75.96 \%$ & $87.13 \%$ & $81.16 \%$ \\
None & $78.52 \%$ & $81.93 \%$ & $80.19 \%$
\end{tabular}

results obtained with a modified matching algorithm including a third rule, which allows a premodified antecedent to match with a definite whose set of premodifiers is a superset of the set of modifiers of the antecedent (an elaboration of rule 2). We tested each of these three heuristics alone and in combination. (The fourth line simply repeats the results shown in Table 7.)

The main result of this evaluation is that using a modified segmentation heuristic (including recency) reduces the overall impact of the heuristics for premodification on the performance of the algorithm in comparison with the system discussed in Vieira and Poesio (1996). The best precision is still achieved by the matching algorithm that does not allow for new information in the anaphoric expression, but the best results overall are again obtained by combining rule 1 and rule 2, although either 2 or 3 works equally well when combined with 1 . (Note that the combination of heuristics 2 and 3 is equivalent to heuristic 3 alone, since rule 3 subsumes rule 2.) Heuristic 2 and 3 alone are counterintuitive and indeed give the poorest results; however, the impact is greater on recall than precision, which suggests that the introduction of new information in noun modification is not very frequent.

One of the problems with our premodifier heuristics is that although a difference in premodification usually indicates noncoreference, as for the company's abrasive segment and the engineering materials segment, there are a few cases in our corpus in which coreferent descriptions have totally different premodification from their antecedents, as in:

the pixie-like clarinetist ... the soft-spoken clarinetist.

These cases would be hard even for a system using real commonsense reasoning, since often the information in the premodifier is new; we consider these examples one of the best arguments for including in the system a focus-tracking mechanism along the lines of Sidner (1979). Our heuristic matching algorithm also suggests wrong antecedents in cases like the rules in (43), when the last mention refers to a modified concept (the new rules are different from the previous ones).

Currently, the rules force executives ...

The rule changes would ...

The rules will eliminate...

Finally, the matching algorithm gets the wrong result in cases such as the population ... the voting population where the new information indicates a subset, superset, or part of a previously mentioned referent. 
Table 10

Evaluation of the heuristics for direct anaphora (Version 1).

\begin{tabular}{lllllll}
\hline Anaphora Classification & $\#$ & + & - & $\mathbf{R}$ & $\mathbf{P}$ & $\mathbf{F}$ \\
\hline Training data & 312 & 243 & 27 & $78 \%$ & $90 \%$ & $83 \%$ \\
Test data & 154 & 103 & 12 & $67 \%$ & $90 \%$ & $77 \%$
\end{tabular}

\begin{tabular}{|c|c|c|c|c|c|}
\hline Anaphora Resolution & \# & + & - & $\mathbf{R}$ & $\mathbf{P}$ \\
\hline Training data & 312 & 237 & 33 & $76 \%$ & $88 \%$ \\
\hline Test data & 154 & 96 & 19 & $62 \%$ & $83 \%$ \\
\hline
\end{tabular}

5.2.4 Overall Results for Anaphoric Definite Descriptions. To summarize, on the basis of the tests just discussed, the heuristics that achieve the best results for anaphoric definite descriptions are:

1. combined loose segmentation and recency,

2. four-sentence window,

3. considering indefinites, definites, and possessives as potential antecedents,

4. the premodification of the description must be contained in the premodification of the antecedent or when the antecedent has no premodifiers.

In Table 10 we present the overall results on anaphora classification and anaphora resolution for the version of the system that does not attempt to resolve bridging descriptions, for both training data and test data. The reason there are different figures for anaphora resolution and classification is that the system may correctly classify a description as anaphoric, but then find the wrong antecedent. We used this set of heuristics when evaluating the heuristics for discourse-new and bridging descriptions in the rest of the paper.

The column headed \# represents the number of cases of descriptions classified as anaphora in the standard annotation; + indicates the total number of anaphora (classification and resolution) correctly identified; - indicates the total number of errors.

5.2.5 Errors in Anaphora Resolution. Before discussing the results of the other heuristics used by the system, we will discuss in more detail some of the errors in the resolution of anaphoric descriptions made by using the heuristics just discussed.

Some errors are simply caused by misspellings in the Treebank, as in the example below, where the antecedent is misspelled as spokewoman.

$$
\text { A Lorillard spokewoman ... The Lorillard spokeswoman }
$$

The most common problems are due to the heuristics limiting the search for antecedents. In (45), both sentence 7 and sentence 30 are outside the window considered by the system when trying to resolve the adjusters in 53 .

(45) 7. She has been on the move almost incessantly since last Thursday, when an army of adjusters, employed by major insurers, invaded the San Francisco area. 
30. Aetna, which has nearly 3,000 adjusters, had deployed about 750 of them

53. Many of the adjusters employed by Aetna and other insurers

Limiting the type of potential antecedents to indefinites, definite descriptions, and possessives, while improving precision, also leads to problems, because the antecedents introduced by other NPs, such as proper names, are missed-e.g., Toni Johnson in (46). The following definite description is then classified by the system as larger situation/unfamiliar. Some of these problems are corrected in Version 2 of the system, which also attempts to handle bridging descriptions and therefore uses algorithms for assigning a type to such entities.

(46) Toni Johnson pulls a tape measure across the front of what was once a stately Victorian home.

The petite, 29-year-old Ms. Johnson ...

The premodification heuristics prevent the system from finding the right antecedent in the (rare) cases of coreferent descriptions with different premodifiers, as in (47).

The Victorian house that Ms. Johnson is inspecting has been deemed unsafe by town officials.

Once inside, she spends nearly four hours measuring and diagramming each room in the 80-year-old house.

In the following example, it is the lack of a proper treatment of postmodification that causes the problem. The system classifies the description the earthquake-related claims as anaphoric to claims from that storm, but it is discourse-new according to the standardized annotation.

(48) Most companies still are trying to sort through the wreckage caused by Hurricane Hugo in the Carolinas last month.

Aetna, which has nearly 3,000 adjusters, had deployed about 750 of them in Charlotte, Columbia, and Charleston.

Adjusters who had been working on the East Coast say the insurer will still be processing claims from that storm through December.

It could take six to nine months to handle the earthquake-related claims.

In (49), the system correctly classifies the definite description the law as anaphoric, but suggests as antecedent an income tax law, whereas a majority of our annotators 
Table 11

Evaluation of the heuristics for identifying discourse-new descriptions.

\begin{tabular}{lllllll}
\hline Discourse-new & $\#$ & + & - & $\mathbf{R}$ & $\mathbf{P}$ & $\mathbf{F}$ \\
\hline Training data & 492 & 368 & 60 & $75 \%$ & $86 \%$ & $80 \%$ \\
Test data & 218 & 151 & 58 & $69 \%$ & $72 \%$ & $70 \%$
\end{tabular}

indicated a money lending law as the antecedent. ${ }^{28}$

Nearly 20 years ago, Mr. Morishita, founder and chairman of Aichi Corp., a finance company, received a 10-month suspended sentence from a Tokyo court for violating a money-lending law and an income tax law.

He was convicted of charging interest rates much higher than what the law permitted, and attempting to evade income taxes by using a double accounting system.

Finally, the system is incapable of resolving plural references to collections of objects introduced by singular NPs, even when these collections were introduced by coordinated noun phrases. Although it would be relatively easy to add rules for handling the simplest cases (possibly at the expense of a decrease in precision), many of these references can only be resolved by means of nontrivial operations.

The owners, William and Margie Hammack, are luckier than many others.

\section{The Hammacks ...}

\subsection{Identification of Discourse-New Descriptions}

The overall recall and precision results for the heuristics for identifying discoursenew descriptions presented in Section 4.2 are shown in Table 11. In this table we do not distinguish between the two types of discourse-new descriptions, unfamiliar and larger-situation (Hawkins 1978). As already mentioned in Section 4.2, distinguishing between the two types of discourse-new descriptions identified by Hawkins, Prince, and others isn't easy even for humans (Fraurud 1990; Poesio and Vieira 1998); and indeed, our heuristics for recognizing discourse-new descriptions work better when evaluated together. The column headed \# represents the number of cases of descriptions classified as discourse-new in the standard annotation; + indicates the total number of discourse-new descriptions correctly identified; - the number of errors. These results are for the version of the system that uses the best version of the heuristics for dealing with anaphoric descriptions discussed above, and that doesn't attempt to resolve bridging descriptions (Version 1 ).

The performance of the specific heuristics discussed in Section 4.2 is shown in Tables 12 to 15 . Table 12 shows the results of the heuristics for larger situation uses on the training data, whereas Table 13 reports the performance on the same data of

28 The law could also be interpreted as referring to "the law system in general," in which case none of the antecedents would be correct (or either could be taken as anchor for a bridging interpretation of the definite). 
Table 12

Evaluation of heuristics for larger situation uses (training data).

\begin{tabular}{lccl}
\hline Larger Situation & Total Found & Errors & Precision \\
\hline Names & 73 & 10 & $86 \%$ \\
Time references & 50 & 7 & $86 \%$ \\
Premodification & 41 & 19 & $54 \%$ \\
Total & 164 & 36 & $78 \%$
\end{tabular}

Table 13

Evaluation of heuristics for unfamiliar uses (training data).

\begin{tabular}{lccl}
\hline Unfamiliar & Total Found & Errors & Precision \\
\hline NP compl/Unexp mod & 32 & 2 & $93 \%$ \\
Apposition & 27 & 2 & $92 \%$ \\
Copula & 8 & 2 & $75 \%$ \\
Postmodification & 197 & 18 & $91 \%$ \\
Total & 264 & 24 & $91 \%$
\end{tabular}

Table 14

Evaluation of heuristics for larger situation uses (test data).

\begin{tabular}{llrl}
\hline Larger Situation & Total Found & Errors & Precision \\
\hline Names & 44 & 14 & $68 \%$ \\
Time references & 21 & 5 & $64 \%$ \\
Premodification & 17 & 9 & $47 \%$ \\
Total & 82 & 28 & $66 \%$
\end{tabular}

the heuristics for unfamiliar uses. We report only precision figures because our standard annotation only gives us information about the classification of these discourse descriptions as discourse-new, not about the reason they were classified in a certain way (larger situation or unfamiliar). The most common feature of discourse-new descriptions is postmodification; the least satisfactory results are those for proper names in premodification. As expected, the heuristics for recognizing unfamiliar uses (many of which are licensed by linguistic knowledge) achieve better precision than those for larger situation uses, which depend more on commonsense knowledge.

Tables 14 and 15 summarize the results of the heuristics for discourse-new descriptions on the test data (Corpus 2). Again, the best results were obtained by the heuristics for recognizing unfamiliar uses. The biggest difference in performance was shown by the heuristic checking the presence of the definite in a copula construction, which performed very well on the training data, but poorly on the test data. The actual performance of that heuristic is difficult to evaluate, however, as a very low recall was reported for both training and test data.

In the following sections, we analyze some of the problems encountered by the version of the system using these heuristics.

Apposition. Coordinated NPs with more than two conjuncts are a problem for this heuristic, since in the Penn Treebank I, coordinated NPs have a structure that matches the pattern used by the system for recognizing appositions. For example, the coordinated NP in the sentence G-7 consists of the U.S., Japan, Britain, West Germany, Canada, 
Table 15

Evaluation of heuristics for unfamiliar uses (test data).

\begin{tabular}{lccl}
\hline Unfamiliar & Total Found & Errors & Precision \\
\hline NP compl/Unexp mod & 16 & 2 & $87 \%$ \\
Apposition & 10 & 2 & $80 \%$ \\
Copula & 6 & 4 & $33 \%$ \\
Postmodification & 95 & 22 & $77 \%$ \\
Total & 127 & 30 & $76 \%$
\end{tabular}

France and Italy has the structure in (51).

$$
\begin{aligned}
& \text { [NP, [NP, the ,U.S.] , , , [NP, Japan] , , , [NP, Britain] , , , [NP, West, } \\
& \text { Germany] , , [NP, Canada], , , [NP, France], and, [NP, Italy]] }
\end{aligned}
$$

Copula. This heuristic was difficult to evaluate because there few examples, and the precision in the two data sets is very different (see Tables 13 and 15 above). One problem is that the descriptions in copula constructions might also be interpreted as bridging descriptions. For instance, the description the result in (52a) below is the result of something mentioned previously, while the copula construction specifies its referent. Other ambiguous examples are (52b) and (52c):

a. The result is that those rich enough to own any real estate at all have boosted their holdings substantially.

b. The chief culprits, he says, are big companies and business groups that buy huge amounts of land not for their corporate use, but for resale at huge profit.

c. The key man seems to be the campaign manager, Mr. Lynch.

Restrictive premodification. One problem with this heuristic is that although proper nouns in premodifier positions are often used with discourse-new definites (e.g., the Iran-Iraq war), they may also be used as additional information in associative or anaphoric uses:

(53) Others grab books, records, photo albums, sofas and chairs, working frantically in the fear that an aftershock will jolt the house again.

$\cdots$

As Ms. Johnson stands outside the Hammack house after winding up her chores there, the house begins to creak and sway.

Restrictive postmodification. If the system fails to find an antecedent or anchor and the description is postmodified, it may wrongly be classified as discourse-new. In (54) the filing on the details of the spinoff was classified as bridging on documents filed ... by the coders, but the system classified it as discourse-new.

Documents filed with the Securities and Exchange Commission on the pending spinoff disclosed that Cray Research Inc. will withdraw the almost $\$ 100$ million in financing it is providing the new firm if Mr. Cray leaves or if the product-design project he heads is scrapped. 
The filing on the details of the spinoff caused Cray Research stock to jump $\$ 2.875$ yesterday to close at $\$ 38$ in New York Stock Exchange composite trading.

Proper nouns. As we have already seen-(46), repeated below as (55)-a definite description that looks like a proper noun (the petite, 29-year-old Ms. Johnson) may in fact be anaphoric. This is not always a problem, as the system does attempt to find antecedents for these definites, as well, but if the antecedent is not found (as in the example below) the description is incorrectly classified as discourse-new.

Toni Johnson pulls a tape measure across the front of what was once a stately Victorian home.

$\cdots$

The petite, 29-year-old Ms. Johnson ...

Special predicates. In this example the system classified as discourse-new a time reference (the same time), which is classified as bridging in the standard annotation.

Newsweek's circulation for the first six months of 1989 was $3,288,453$, flat from the same period last year.

U.S. News' circulation in the same time was $2,303,328$, down $2.6 \%$.

\subsection{Bridging Descriptions}

As mentioned in Section 2, our corpus annotation experiments showed bridging descriptions to be the most difficult class for humans to agree on. Even when our annotators agreed that a particular expression was a bridging description, different anchors would be available in the text for the interpretation of that bridging description. This makes the results of the system for this class very difficult to evaluate; furthermore, the results must be evaluated by hand.

We first tested the heuristics individually on the training data (the same data used in a previous analysis of the performance of our system on bridging descriptions [Vieira and Teufel 1997]) by adding them to Version 1 of the system one at a time. These separate tests were manually evaluated. We then integrated all of these heuristics into a version of the system called Version 2, using both automatic and manual evaluation. In this section we discuss only the results of the individual heuristics; the overall results of Version 2 are discussed in Section 6.

Bridging descriptions are much more sensitive than other types of definite descriptions to the local focus (Sidner 1979); for this reason, Version 2 uses a different search strategy for bridging descriptions than for other definite descriptions. Rather than considering all definite descriptions in the current window simultaneously, it goes back one sentence at a time and stops as soon as a relation with a potential anchor is found.

5.4.1 Using WordNet to Identify Anchors. Our system consults WordNet to determine if a definite description may be semantically related to one of the NPs in the previous 
Table 16

Evaluation of the search for anchors using WordNet.

\begin{tabular}{lccc}
\hline Bridging Class & Relations Found & Right Anchors & \% Right \\
\hline Synonymy & 11 & 4 & $36 \%$ \\
Hyponymy & 59 & 18 & $30 \%$ \\
Meronymy & 6 & 2 & $33 \%$ \\
Sister & 30 & 6 & $20 \%$ \\
Total & 106 & 30 & $28 \%$
\end{tabular}

five sentences. ${ }^{29}$ The results of this search over our training corpus, in which 204 descriptions were classified as bridging, are shown in Table 16. It is interesting to note that the semantic relations found in this automatic search were not always those observed in our manual analysis.

The main reason the figures are so low is that the existence of a semantic relation in WordNet is not a sufficient condition (nor a strong indication) to establish a link between an antecedent and a bridging description. In only about a third of the cases was a potential antecedent for which we could find a semantic relation in WordNet an appropriate anchor. An example is (57): although there is a semantic relation between argument and information in WordNet, the description the argument is related to the VP contend rather than to the NP information. Some form of focusing seems to play a crucial role in restricting the range of antecedents (see also the discussion in Hitzeman and Poesio [1998]).
A SEC proposal to ease reporting requirements for some company executives would undermine the usefulness of information on insider trades as a stock-picking tool, individual investors and professional money managers contend.
They make the argument in letters to the agency about rule changes proposed this past summer that, among other things, would exempt many middle-management executives from reporting trades in their own companies' shares.

Sense ambiguity is responsible for some of the false positives. For instance, the noun company has at least two distinct senses: "visitor" (as in I have company) and "business." A relation of hypernymy was found between company and human (its "visitor" sense), whereas in the text the noun company was used in the "business" sense. A more important problem, however, is the incompleteness of the information encoded in WordNet. To have an idea of how complete the information in WordNet is concerning the relations that are encoded, we selected from our two corpora 70 bridging descriptions that we had manually identified as being linked to their anchors by one of the semantic relations encoded in WordNet-synonymy, hypernymy (hyponymy), and meronymy (holonymy). In Table 17 we show the percentages of such relations actually encoded in WordNet. (The fourth column in the table indicates the cases in which the expected relation is not encoded, but the two nouns are sisters in the hierarchy.)

As we can see from the table, the recall figure was quite disappointing, especially for synonymy relations. In some cases, the problem was simply that some of the

29 We found that for bridging descriptions, a five-sentence window worked bctter than a four-sentence one. 
Table 17

Evaluation of the encoding of semantic relations in WordNet.

\begin{tabular}{llcll}
\hline Bridging Class & Anchor/DD Pairs & Found in WN & Found Sister & $\%$ \\
\hline Syn & 20 & 5 & 2 & $35 \%$ \\
Hyp & 32 & 17 & 1 & $56 \%$ \\
Mer & 18 & 5 & 2 & $38 \%$ \\
Total & 70 & 27 & 5 & $46 \%$
\end{tabular}

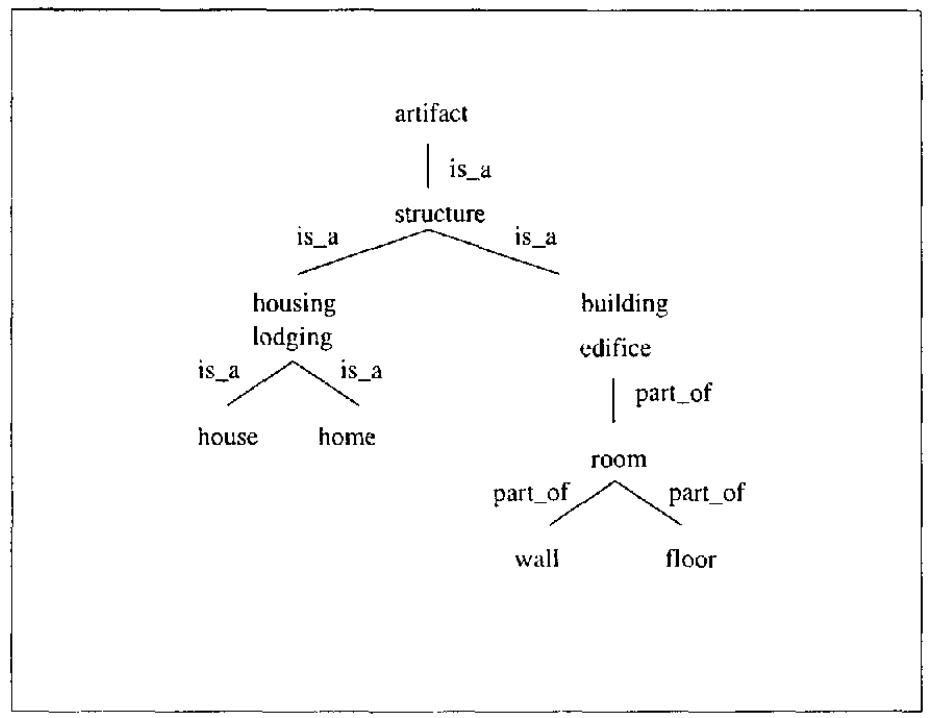

Figure 2

An example of problematic organization in WordNet.

words we looked for were not in WordNet: examples include newsweekly (news-weekly), crocidolite, countersuit (counter-suit). Other times, the word we looked for was contained in WordNet, but not in the same typographic format as it was presented in the text; for example we had spinoff in a text, whereas WordNet had only an entry for spinoff. A second source of problems was the use in the WSJ articles of domain-specific terminology with context-dependent senses, such as slump, crash, and bust, which in articles about the economy are all synonyms. Finally, in other cases the relations were missing due to the structure of WordNet: for instance, in WordNet the nouns room, wall, and floor are encoded as part of building but not of house (see Figure 2).

In summary, our tests have shown that the knowledge encoded in WordNet is not sufficient to interpret all semantic relations between a bridging description and its antecedent found in the kind of text we are dealing with: only $46 \%$ of the relations observed were encoded in WordNet. The possibility of using domain-specific, automatically acquired lexical information for this purpose is being explored: see, for example, Poesio, Schulte im Walde, and Brew (1998). In addition, we found that just looking for the closest semantic relative is not enough to find anchors for bridging descriptions; this search has to be constrained by some type of focusing mechanism.

5.4.2 Evaluating the Results for Bridging Descriptions Based on Proper Names. Identifying named entity types is a prerequisite for resolving descriptions based on names. The simple heuristics discussed in Section 5.4 identified entity types for $66 \%$ 
(535/814) of all names in the corpus (organizations, persons, and locations); precision was $95 \%{ }^{30}$ The errors we found were sometimes due to name or sense ambiguity. In the same text a name may refer both to a person and a company, as in Cray Computers Corp. and Seymour Cray. When looking in WordNet for a type for the name Steve Reich we found for the name Reich the type country. These problems have also been noted by the authors of systems participating in MUC-6 (Appelt 1995). We also found undesirable relations such as hypernymy for person and company.

\subsubsection{Evaluating the Results for Bridging Descriptions Based on Compound Nouns.} We had 25 definite descriptions manually identified as based on compound nouns. For these 25 cases, our implemented heuristics achieved a recall of $36 \%(9 / 25)$ but, in some cases, found valid relations other than the ones we identified. The low recall was sometimes due to segmentation. Sometimes the spelling of the premodification was slightly different from the one of the description, as in a 15-acre plot ... the 15 acres.

\section{Overall Evaluation of the System}

As mentioned above, we implemented two versions of the system. Version 1 only resolves direct anaphora and identifies discourse-new descriptions; Version 2 also deals with bridging descriptions. Both versions of the system have at their core a decision tree in which the heuristics discussed in the previous sections are tried in a fixed order to classify a certain definite description and find its anchor. Determining the optimal order of application of the heuristics in the decision tree is crucial to the performance of the system. In both versions of the system we used a decision tree developed by hand on the basis of extensive evaluation; we also attempted to determine the order of application automatically, by means of decision tree learning algorithms (Quinlan 1993).

In this section we first present the hand-crafted decision tree and the results obtained using this decision tree for Version 1 and Version 2; we then present the results concerning the agreement between system and annotators, and we briefly discuss the results obtained using the decision tree acquired automatically.

\subsection{Integration of the Heuristics}

The hand-crafted order of the heuristics in both versions is the following. For each NP of the input,

1. The system assigns an index to it.

2. The NPs that may serve as potential antecedents are made available for description resolution by means of the optimal selection criterion discussed in Section 4.1.

30 By comparison, the systems participating in MUC-6 had a recall for the named entity task ranging from $82 \%$ to $96 \%$, and precision from $89 \%$ to $97 \%$, but used comprehensive lists of cue words or consulted dictionaries of names. The system from Sheffield (Gaizauskas et al. 1995), for instance, used a list of 2,600 names of organizations, 94 company designators (Co., Ltd, PLC, etc.), 160 titles (Dr., Mr., etc.), about 500 human names from the Oxford Advanced Learner's Dictionary, 2,268 place names (country, province, and city names), and other trigger words for locations, government institutions and organizations (Golf, Mountain, Agency, Ministry, Airline, etc.). In MUC-7, the best combined precision score, 93.39\%, was achieved by the system from LTG in Edinburgh (Mikheev, Moens, and Grover 1999), which doesn't use such knowledge sources. We used this system in a version of our prototype that only attempts to resolve bridging descriptions (Ishikawa 1998). 
3. If the NP is a definite description, the system applies to it the following tests. The first test passed by the definite (if any) determines its classification, and after that, the next NP is processed.

a. Examine a list of special predicates in order to identify some of the unfamiliar and larger situation uses (see Section 4.2).

b. Check whether the definite NP occurs in an appositive construction; if so, there is no need to find an antecedent for it. The NP is classified as discourse-new, unfamiliar use.

c. Try to find an antecedent for the definite description among the antecedents that are accessible according to combined loose segmentation and recency, by matching head nouns and dealing with premodification and postmodification (see Section 4.1 and Section 5.2). If the system succeeds, the description is classified as direct anaphora and the relation of coreference between the two NP indexes is asserted.

d. Verify if the head of the NP is a proper noun (by checking whether it is capitalized). If so, the description is classified as discourse-new, larger situation use.

e. Check if the definite has a restrictive postmodifier. Definites that are not anaphoric and have restrictive postmodifiers are classified as discourse-new, unfamiliar uses.

f. Check if there is a proper noun in premodifier position; if so, the definite description is classified as discourse-new, larger situation use.

g. Check if the definite occurs in a copula construction. If so, the description is classified as discourse-new, unfamiliar use.

h. If the tests above failed, Version 1 of the system stops; Version 2 starts searching for an anchor going backwards one sentence at a time and according to the following heuristics (in this order):

i. proper names

ii. compound nouns

iii. WordNet look-up

If one of the three tests above succeeds the description is classified as bridging and the association between description and anchor indexes is asserted.

The decision tree encoded by this algorithm is shown in Figure 3.

Note that before trying to find an antecedent, the system executes a few tests for identifying discourse-new descriptions; in other words, the strategy adopted is:

- first eliminate some nonanaphoric cases using "safe" heuristics (first two tests); $;^{31}$

- if that fails, try to find a same-head antecedent (third test);

- if that doesn't work either, look for an indication that the description is discourse-new (following four tests),

31 We considered special predicates and appositions as reliable indications of discourse novelty; in addition, definite descriptions that matched these patterns produced errors in anaphora resolution which were eliminated by processing them first. 


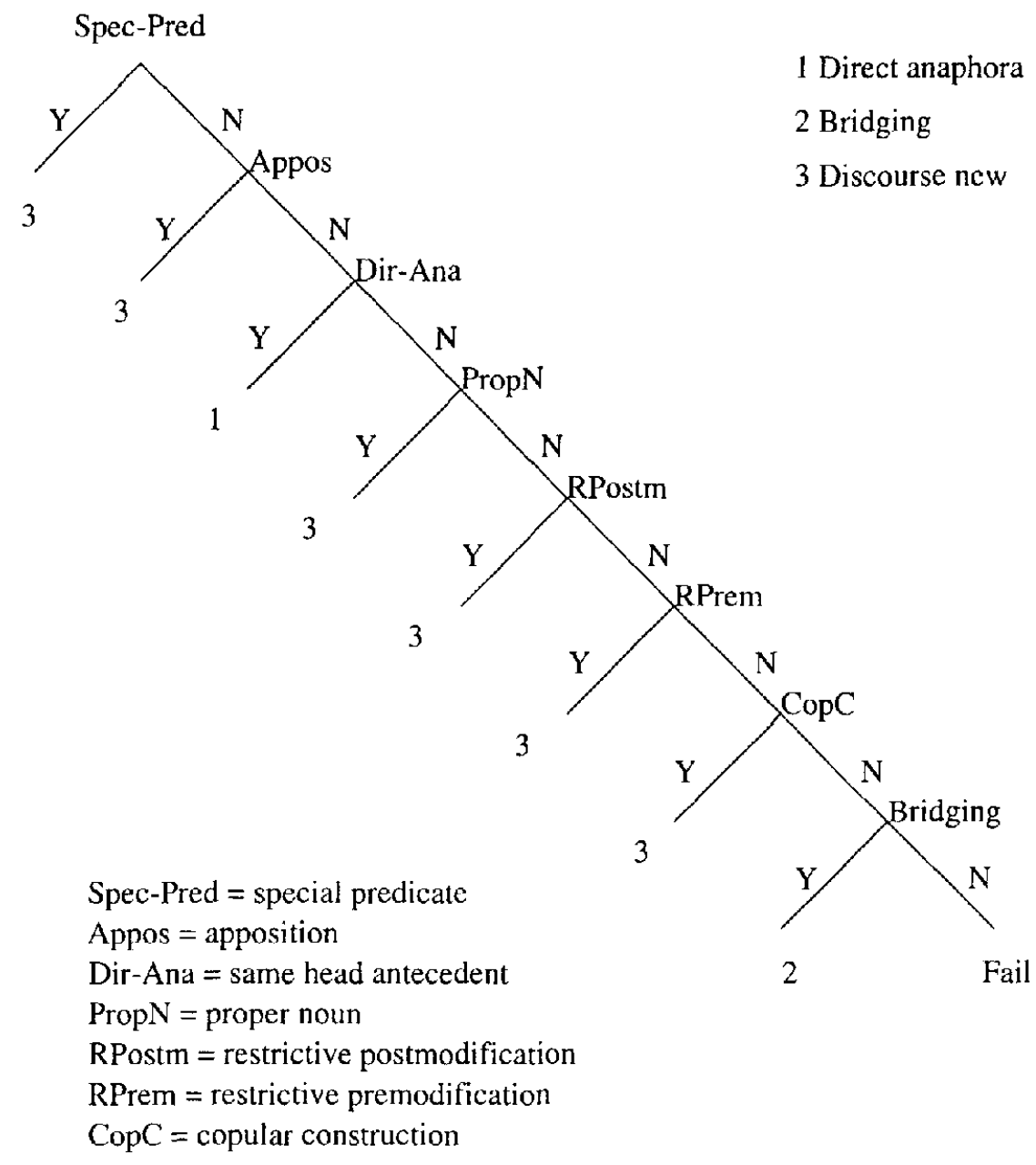

Figure 3

Hand-designed decision tree for Version 1 and Version 2.

- only then try to interpret the definite description as a bridge (last test).

The heuristics for recognizing bridging descriptions are only applied when the other heuristics fail. This is because the performance of these heuristics is very poor and also because some of the heuristics that deal with bridging descriptions are computationally expensive; the idea was to eliminate those cases less likely to be bridging before applying these heuristics. The system does not classify all occurrences of definite descriptions: when none of the tests succeeds, the definite description is not classified. We observed in our first tests that definite descriptions not resolved as direct anaphora and not identified as discourse-new by our heuristics were mostly classified in the standardized annotation as bridging descriptions or discourse-new. Examples of discourse-new descriptions not identified by our heuristics are larger situation uses such as the zorld, the nation, the government, the economy, the marketplace, the spring, the 


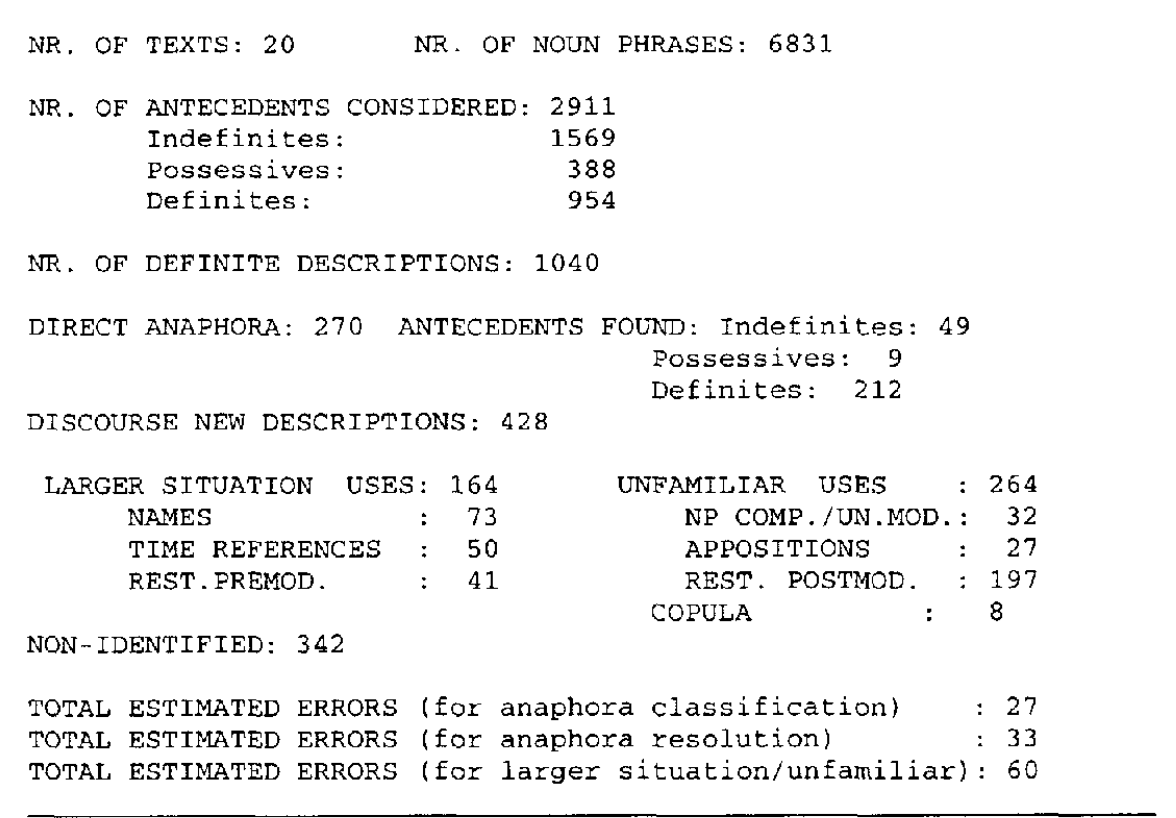

\section{Figure 4}

Global results of Version 1 on the training data.

other hand, the spot, and the 1920s, or discourse-new NPs with restrictive premodification such as the low $40 \%$ range, the defense capital good sector, the residential construction industry, the developing world, and the world-wide supercomputer market.

\subsection{Results for Anaphora and Discourse-New Descriptions}

We present below the overall results of the version of our system dealing with direct anaphora and discourse-new descriptions only (Version 1).

Training Data. The output of the optimal configuration of Version 1 for the training data is shown in Figure 4. A total of 20 texts were processed, containing 6,831 NPs. Almost half of these NPs $(2,911)$ were considered as potential antecedents; 1,040 descriptions were processed by the system. An antecedent was identified for 270 of them; for 212 out of the 270 definite descriptions classified as anaphoric same-head by the system, the antecedent was a definite NP. According to the annotation of one of our coders (not the system's output), the 312 anaphoric descriptions were grouped in 164 coreference chains and 86 of these chains were initiated by definite descriptions.

In Figure 5, the results reported by the system are compared with the standard annotation. The figure also shows how descriptions which were not resolved by the system were classified in the standard annotation. Most of the descriptions not classified by the system were bridging descriptions.

The overall precision and recall results of Version 1 of the system are shown in Table 18. Note that because a large number of definite descriptions are not classified, the overall recall is only $59 \%$, even though the recall for both anaphoric and discoursenew descriptions is much higher.

Test Data. Next, the system was evaluated using the test data, Corpus 2, which had not been used to develop the heuristics. The results are shown in Figures 6 and 7 . 


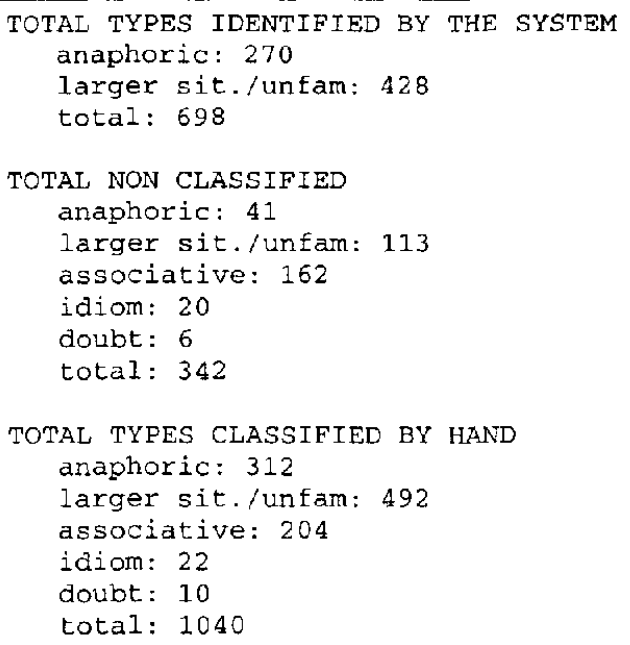

\section{Figure 5}

Summary of the results of Version 1 on training data.

\section{Table 18}

Global results of Version 1 on training data.

\begin{tabular}{llll}
\hline System's tasks & $\mathbf{R}$ & $\mathbf{P}$ & $\mathbf{F}$ \\
\hline Anaphora classification & $78 \%$ & $90 \%$ & $83 \%$ \\
Anaphora resolution & $76 \%$ & $88 \%$ & $81 \%$ \\
Discourse-new & $75 \%$ & $86 \%$ & $80 \%$ \\
Overall & $59 \%$ & $88 \%$ & $70 \%$
\end{tabular}

\section{Table 19}

Evaluation of Version 1 on the test data.

\begin{tabular}{llll}
\hline System's tasks & $\mathbf{R}$ & $\mathbf{P}$ & $\mathbf{F}$ \\
\hline Anaphora classification & $67 \%$ & $90 \%$ & $77 \%$ \\
Anaphora resolution & $62 \%$ & $83 \%$ & $71 \%$ \\
Discourse-new & $69 \%$ & $72 \%$ & $70 \%$ \\
Overall & $53 \%$ & $76 \%$ & $63 \%$
\end{tabular}

The recall and precision figures for the system's performance over the test data are presented in Table 19. This corpus consisted of 14 texts, containing 2,990 NPs. Again, almost half of the NPs were considered as potential antecedents. The system processed 464 definite descriptions; of these, the system could classify 324: 115 as direct anaphora, 209 as discourse-new. Of the antecedents, 88 were definites themselves. The system incorrectly resolved 77 definite descriptions: 19 anaphoric definites and 58 discoursenew. As before, there were just a few more errors in anaphora resolution than in anaphora classification. The overall recall for the test data was 53\% (247/464); precision was $76 \%(247 / 324)$.

One difference between the results on the two data sets is the distribution into classes of those descriptions that the system fails to classify. In the first corpus, the largest number of cases not classified are bridging descriptions. By contrast, the largest number of cases not classified by the system in Corpus 2 are discourse-new. 


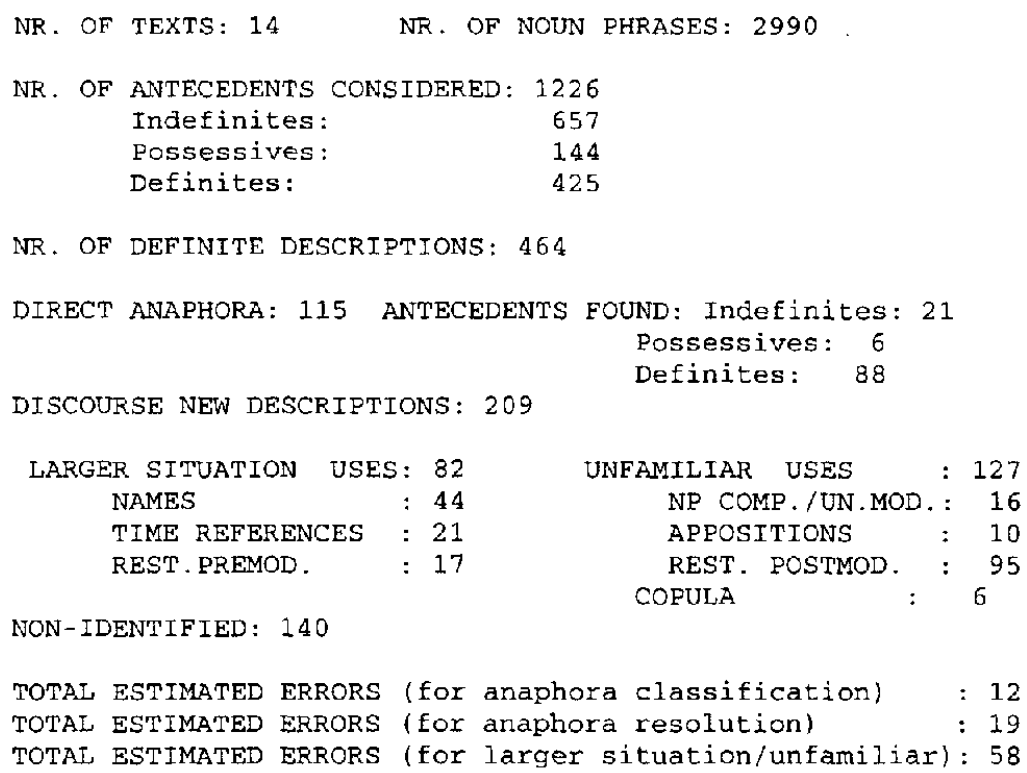

NR. OF DEFINITE DESCRIPTIONS: 464

DIRECT ANAPHORA: 115 ANTECEDENTS FOUND: Indefinites: 21

Possessives: 6

Definites: 88

DISCOURSE NEW DESCRIPTIONS: 209

\section{Figure 6}

Global results of Version 1 on test data.

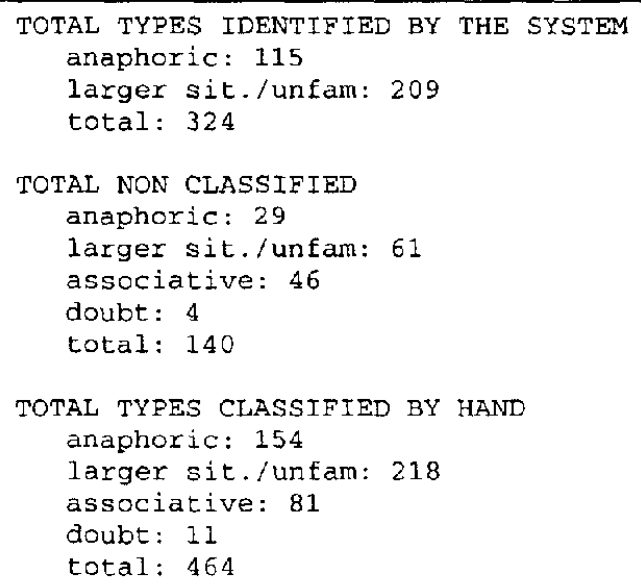

Figure 7

Summary of the results for test data.

\subsection{Results for Bridging Descriptions}

As discussed in Section 5.4, the results of the heuristics for bridging descriptions presented in Section 4.3 were not very good. We nevertheless included these heuristics in Version 2 of the system, which, as discussed above, applied them to those descriptions that failed to be recognized as direct anaphora or discourse-new. The heuristics were applied in the following order:

1. proper names, 
Table 20

Evaluation of the bridging heuristics all together.

\begin{tabular}{llc}
\hline $\begin{array}{l}\text { Bridging } \\
\text { Class }\end{array}$ & Found by System & $\begin{array}{l}\text { False } \\
\text { Positive }\end{array}$ \\
\hline Names & 12 & $\mathbf{1 4}$ \\
Common nouns & 15 & 10 \\
WordNet & 34 & 76 \\
Total & 61 & 100
\end{tabular}

Table 21

Comparative evaluation of the two versions (test data).

\begin{tabular}{llll}
\hline System's versions & $\mathbf{R}$ & $\mathbf{P}$ & $\mathbf{F}$ \\
\hline V.1 Overall & $53 \%$ & $76 \%$ & $62 \%$ \\
V.2 Overall & $57 \%$ & $70 \%$ & $62 \%$
\end{tabular}

2. compound nouns,

3. WordNet,

Training Data. The manual evaluation of the results of Version 2 on the training data is presented in Table 20. The table lists the number of acceptable anchors and the number of false positives found by each heuristic. Note that the system sometimes finds anchors that are not those identified manually, but are nevertheless acceptable.

We found fewer bridging relations than the number we observed in the corpus analysis (204); furthermore, the number of false positives produced by such heuristics is almost twice the number of right answers.

Test Data. Version 2 was tested over the test data using automatic evaluation-i.e., the system was only evaluated as a classifier, and the anchors found were not analyzed. A total of 57 bridging relations were found, but only 19 of the definite descriptions classified as bridges by the system had been classified as bridging descriptions in the standard annotation. Compared to Version 1 of the system, which does not resolve bridging descriptions, Version 2 has higher recall but lower precision, as shown in Table 21 .

\subsection{Agreement among System and Annotators for Version 1 and Version 2}

As a second form of evaluation of the performance of the system, we measured its agreement with the annotators on the test data using the $K$ statistic.

Version 1 of the system finds a classification for 318 out of 464 definite descriptions in Corpus 2 (the test data). If all the definite descriptions that the system cannot classify are treated as discourse-new, the agreement between the system and the three subjects that annotated this corpus on the two classes first-mention (= discourse-old) and subsequent-mention (= discourse-new or bridges) is $K=0.7$; this should be compared with an agreement of $K=0.77$ between the three annotators themselves. If, instead of counting these definite descriptions as discourse-new, we simply do not include them in our measure of agreement, then the agreement between the system and the annotators is $K=0.78$, as opposed to $K=0.81$ between the annotators. (Notice that the fact that the agreement between annotators goes up, as well, indicates that the definite descriptions that the system can't handle are "harder" than the rest.) 
Version 2 finds a classification for 355 out of 464 definite descriptions; however, its agreement figures are worse. If we count the cases that the system can't classify as discourse-new, the agreement between the system and the three annotators for the three classes is $K=0.57$; if we count them as bridges, $K=0.63$; if we just discard those cases, $K=0.63$ again. (By comparison, the agreement among annotators on the three classes was $K=0.68$ overall and $K=0.70$ on just the cases that the system was able to classify.) As mentioned above, the cases that the system can't handle are mainly discourse-new descriptions (see Figure 7).

\subsection{Deriving the Order of Application of the Heuristics Automatically}

6.5.1 Inducing a Decision Tree. The decision tree discussed in Section 6.1 was derived manually, by trial and error. We also tried to derive the order of application of the heuristics automatically. To do this, we used a modified version of the system to assign Boolean feature values to each definite description in the training corpus (i.e., the system checked if the features applied to a definite description instance or not). The following features were used:

1. Special predicates (Spec-Pred): this feature has the value yes if a special predicate occurs in the definite description (as specified in Section 4.2), and if a complement is there when needed.

2. Direct anaphora (Dir-Ana): this feature has the value yes if the system can find an antecedent with a same-head noun for that description (respecting the constraints discussed in Section 4.1).

3. Apposition (Appos): yes when the description is in appositive construction.

4. Proper noun (PropN): yes when the description has a capitalized initial.

5. Restrictive postmodification (RPostm): yes if the definite description is modified by relative or associative clauses.

This list of features, together with the classification assigned to each description in the standard annotation (DDUse), was used to train an implementation of Quinlan's learning algorithm ID3 (Quinlan 1993). We excluded the verification of restrictive premodification and copula constructions, since these parameters had given the poorest results before (see Section 6.2). An example of the samples used to train ID3 is shown in (58).

$\begin{array}{cccclc}\text { Spec-Pred } & \text { Dir-Ana } & \text { Appos } & \text { PropN } & \text { RPostm } & \text { DDUse } \\ \text { no } & \text { no } & \text { no } & \text { yes } & \text { no } & 3 \\ \text { no } & \text { no } & \text { no } & \text { no } & \text { yes } & 3 \\ \text { no } & \text { no } & \text { no } & \text { no } & \text { no } & 2 \\ \text { no } & \text { no } & \text { no } & \text { no } & \text { no } & 2 \\ \text { no } & \text { no } & \text { no } & \text { no } & \text { no } & 1 \\ \text { no } & \text { yes } & \text { no } & \text { no } & \text { no } & 1\end{array}$

The algorithm generates a decision tree on the basis of the samples given. The resulting decision tree is presented in Figure 8.

The main difference between this algorithm and the algorithm we arrived at by hand is that the first feature checked by the decision tree generated by ID3 is the presence of an antecedent with a same-head noun. The presence of special predicates, which we adopted as the first test in our decision tree, is only the fourth test in the tree in Figure 8. 


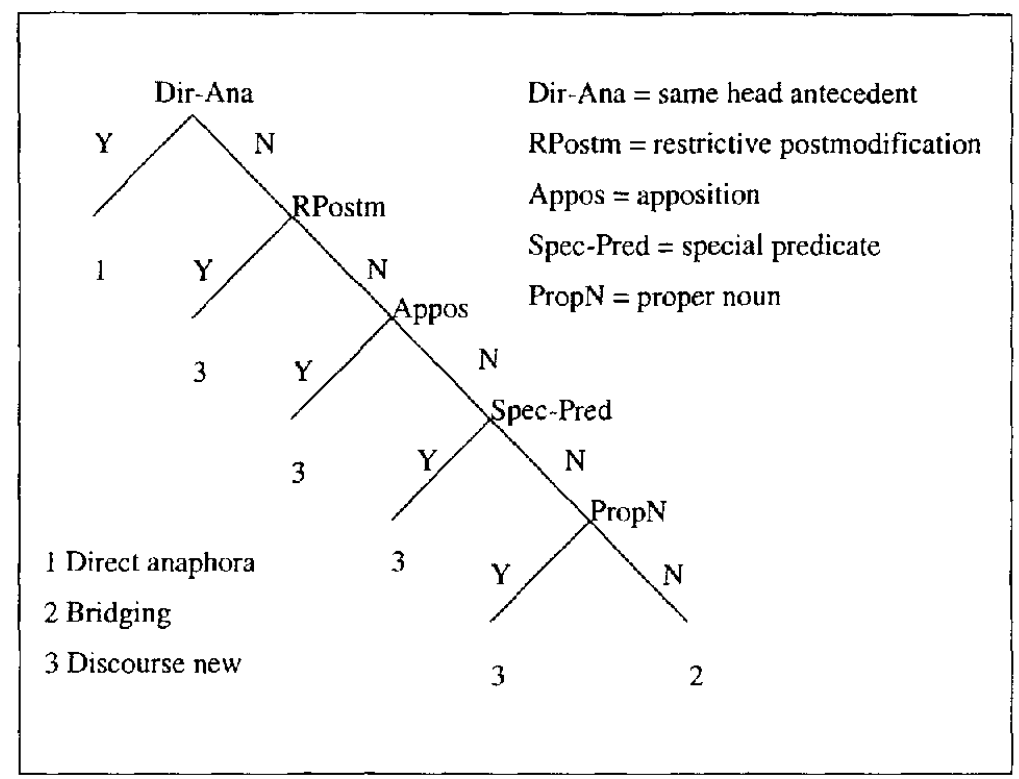

Figure 8

Generated decision tree.

6.5.2 Evaluation of the Automatically Learned Decision Tree. The performance of the learned decision tree was compared with that of the algorithm we arrived at by trial and error as follows: The first 14 texts of Corpus 1 ( 845 descriptions) were used as training data to generate the decision tree. We then tested the learned algorithm over the other 6 texts of that corpus (195 instances of definite descriptions).

Two different tests were undertaken:

- first, we gave as input to the learning algorithm all cases classified as direct anaphora, discourse-new, or bridging, 818 in total (this test produces the decision tree presented in the previous section);

- in a second test, the algorithm was trained only with direct anaphora and discourse-new descriptions (639 descriptions); all cases classified as bridging, idiom, or doubt in the standard annotation were not given as input in the learning process. This algorithm was then only able to classify descriptions as one of those two classes. The resulting decision tree classifies descriptions with a same-head antecedent as anaphoric; all the rest as discourse-new.

Here we present the results evaluated all together, considering the system as a classifier only, i.e., without considering the tasks of anaphora resolution and of identification of discourse-new descriptions separately. The output produced by the learned algorithm is compared to the standard annotation. Since the learned algorithm classifies all cases, the number of responses is equal to the number of cases, as a consequence, recall is the same as precision, and so is the $F$ measure.

The tests over 6 texts with 195 definite descriptions gave the following results:

- $R=P=F=69 \%$ when the algorithm was trained with three classes;

- $R=P=F=75 \%$, when training with two classes only. 
The best results were achieved by the algorithm trained for two classes only. This is not surprising, especially considering how difficult it was for our subjects to distinguish between discourse-new and bridging descriptions.

The hand-crafted decision tree (Version 2 ) achieved $62 \%$ recall and $85 \%$ precision ( $F=71.70 \%$ ) on those same texts: i.e., a higher precision, but a lower $F$ measure, due to a lower recall, since-unlike the learned algorithm-it does not classify all instances of definite descriptions. If, however, we take the class discourse-new as a default for all cases of definite descriptions not resolved by the system, recall, precision, and $F$ value go to $77 \%$, slightly higher than the rates achieved by the decision tree produced by ID3.

As the learned decision tree has the search for a same-head antecedent as the first test, we modified our algorithm to work in the same way, and tested it again with the two corpora. The results with this configuration were:

- $R=0.75, P=0.87, F=0.80$, for the training data (compared with $R=0.76, P=0.88, F=0.81$ ) ;

- $R=0.59, P=0.83, F=0.69$, for the test data (compared with $R=0.62$, $P=0.83, F=0.71)$.

In other words, the results were about the same, although a slightly better performance was obtained when the tests to identify discourse-new descriptions were tried first.

\section{Other Computational Models of Definite Description Processing}

A major difference between our proposal and almost all others (theoretical and implemented) is that we concentrate on definite descriptions; most of the systems we discuss below attempt to resolve all types of anaphoric expressions, often concentrating on pronouns. Focusing on definite descriptions allowed us to investigate what types of lexical knowledge and commonsense inference are actually used in natural language comprehension.

From an architectural standpoint, the main difference between our work and other proposals in the literature is that we paid considerably more attention to the problem of identifying discourse-new definite descriptions. ${ }^{32}$

Previous work on computational methods for definite description resolution can be divided in two camps: proposals that rely on commonsense reasoning (and are therefore either mainly theoretical or domain dependent), and systems that can be quantitatively evaluated, such as those competing on the coreference task in the Sixth and Seventh Message Understanding Conference (Sundheim 1995). We discuss these two types of work in turn.

\subsection{Models Based On Commonsense Reasoning}

The crucial characteristic of these proposals is that they exploit hand-coded commonsense knowledge, and cannot therefore be tested on just any arbitrary text. Some of them are simply tested on texts that were especially built for the purpose of testing the system (Carter 1987; Carbonell and Brown 1988); systems like the Core Language Engine are more robust, but they have to be applied to a domain restricted enough that all relevant knowledge can be encoded by hand.

32 This problem is also a central concern in the work by Bean and Riloff (1999). 
Sidner's Theory of Definite Anaphora Comprehension. In her dissertation, Sidner (1979) proposed a complete theory of definite NP resolution, including detailed algorithms for resolving pronouns, anaphoric definite descriptions, and bridging descriptions. She also proposed methods for resolving larger situation uses; the one class her methods do not handle are those definite descriptions that, following Hawkins, we have called unfamiliar uses.

The main contribution of Sidner's dissertation is her theory of focus and its role in resolving definite NPs; to this day, her focus-tracking algorithms are arguably the most detailed account of the phenomenon. The main problem with Sidner's work from our perspective is that her algorithms rely heavily on the availability of a semantic network and causal reasoner; furthermore, some of the inference mechanisms are left relatively underspecified (this latter problem was in part corrected in subsequent work by Carter-see below). Lexical and commonsense knowledge play three important roles in Sidner's system: they are used to track focus, to resolve bridging descriptions and larger situation uses, and to evaluate interpretive hypotheses, discarding those that seem implausible. Only recently have robust knowledge-based methods for some of these tasks begun to appear, and their performance is still not very good, as seen above in our discussion of using WordNet as a semantic network; ${ }^{33}$ as for checking the plausibility of a hypothesis on the basis of causal knowledge about the world, we now have a much better theoretical grasp of how such inferences could be made (see, for example, Hobbs et al. [1993] and Lascarides and Asher [1993]), but we are still quite a long way from a general inference engine.

We also found that some of Sidner's resolution rules are too restrictive. For example, her Cospecification rule 1 prescribes that definite description and focus must have the same head, and no new information can be introduced by the definite; but this rule is violated fairly frequently in our corpus. This criticism is not new: In 1983, it was already recognized that an anaphoric full noun phrase may include some new and unshared information about a previously mentioned entity (Grosz, Joshi, and Weinstein 1983), and Carter (1987) weakened some of the restrictions proposed by Sidner in his system.

Carter's Shallow Processing Anaphor Resolver. Carter (1987) implemented a modified version of Sidner's algorithm and integrated it with an implemented version of Wilks' theory of commonsense reasoning. This work is interesting for two reasons: first of all, because Carter, unlike Sidner, attempted to evaluate the performance of his system; and because, in doing so, he addressed the commonsense reasoning problem in some detail.

Carter's system, SPAR, is based on the Shallow Processing Hypothesis: that in resolving anaphors, reasoning should be avoided as much as possible. This is, of course, the same approach taken in our own work, which could be seen as pushing Carter's approach to the extreme. The difference is that when it becomes necessary, SPAR does use two commonsense knowledge sources: a semantic network based on Alshawi's theory of memory for text interpretation (Alshawi 1987) and a causal reasoner based on Wilks' work (Wilks 1975). In both cases, the necessary information was encoded by hand.

Carter's system was tested over short stories specifically designed for the testing of the system: about 40 written by Carter himself, and 23 written by others. These latter contain about 80 definite descriptions. SPAR correctly resolved all anaphors in the stories written by Carter, and 66 out of 80 of the descriptions in the 23 other stories.

33 An implementation of a (simplified) version of Sidner's focus-tracking algorithms capable of being used by a system like ours was presented in Azzam, Humphreys, and Gaizauskas (1998). 
(Carter himself points out that these results are "of limited significance because of the simplicity of the texts processed compared to 'real' texts" [p. 238].)

The Core Language Engine. The Core Language Engine (CLE) (Alshawi 1992) is a domainindependent system developed at SRI Cambridge, which translates English sentences into formal representations. The system was used by SRI for a variety of applications, including spoken language translation and airline reservations. The CLE makes use of a core lexicon (to which new entries can be added) and uses an abductive commonsense reasoner to produce an interpretation and to verify the plausibility of choice of referents from an ordered list; the required world knowledge has to be added by hand for each domain, together with whatever lexical knowledge is needed.

The construction of the formal representation goes through an intermediate stage called quasi-logical form (QLF). The QLF may contain unresolved terms corresponding to anaphoric NPs including, among others, definite descriptions. The resolution process that transforms QLFs into resolved logical form representations of sentences is described in Alshawi (1990). Definite descriptions are represented as quantified terms. The referential readings of definite descriptions are handled by proposing referents from the external application context (larger situation uses) as well as the CLE context model (anaphoric uses). Attributive readings may also be proposed during QLF resolution; some of these seem to correspond to our unfamiliar uses. Thus, the CLE seems to account for discourse-new descriptions, although they are not explicitly mentioned, and the methods used for choosing a referential or an attributive interpretation are not discussed. To our knowledge, no analysis of the performance of the system has been published.

\subsection{The Systems Involved in the MUC-6 Coreference Task}

The seven systems that participated in the MUC-6 competition can all be quantitatively evaluated; they achieved recall scores ranging from $35.69 \%$ to $62.78 \%$ and precision scores ranging from $44.23 \%$ to $71.88 \%$ on nominal coreference.

It is important to note that the evaluation in MUC-6 differed from ours in three important aspects. First of all, these systems have to parse the texts, which often introduces errors; furthermore, these systems often cannot get complete parses for the sentences they are processing. Secondly, the evaluation in MUC- 6 considers the coreferential chain as a whole, and not only one correct antecedent. The third difference is that these systems process a wider range of referring expressions, including pronouns and bare nouns, while our system only processes definite NPs. On the other hand, not all definite descriptions are marked in the MUC- 6 coreference task: these systems are only required to identify identity relations, and only if the antecedent was introduced by a noun phrase (not if it was a clause or a conjoined NP). This leaves out discoursenew descriptions and, especially, bridging descriptions, which, as we have seen, are by far the most difficult cases.

Kameyama (1997) analyzes in detail the coreference module of the SRI system that participated in MUC-6 (Appelt et al. 1995). This system achieved one of the top scores for the coreference task: a recall of $59 \%$ and a precision of $72 \%$. The SRI system uses a sort hierarchy claimed to be sparse and incomplete. For definite descriptions, Kameyama reports the results of a test on five articles, containing 61 definite descriptions in total; recall was $46 \%(28 / 61)$, and for proper names, $69 \%(22 / 32)$. The precision figures for these two subclasses are not reported. Some of the errors in definite descriptions are said to be due to nonidentity referential relations; however, there is no mention of differences between discourse-new and bridging descriptions. Other errors were said to be related to failure in recognizing synonyms. 


\subsection{Probabilistic Methods in Anaphora Resolution}

Aone and Bennet (1995) propose an automatically trainable anaphora resolution system. They train a decision tree using the $C 4.5$ algorithm by feeding feature vectors for pairs of anaphor and antecedent. They use 66 features, including lexical, syntactic, semantic, and positional features. Their overall recall and precision figures are $66.56 \%$ and $72.18 \%$. Considering only definite NPs whose referent is an organization (that is the only distinction available in their report), recall is $35.19 \%$ and precision $50 \%$ (measured on 54 instances). Their training and test texts were newspaper articles about joint ventures, and they claim that because each article always talked about more than one organization, finding the antecedents of organizational anaphora was not straightforward.

In Burger and Connolly (1992) a Bayesian network is used to resolve anaphora by probabilistically combining linguistic evidence. Their sources of evidence are ccommand (syntactic constraints), semantic agreement (gender, person, and number plus a term subsumption hierarchy), discourse focus, discourse structure, recency, and centering. Their methods are described and exemplified but not evaluated. A Bayesian framework is also proposed by Cho and Maida (1992) for the identification of definite descriptions' referents.

\section{Conclusions and Future Work}

\subsection{Contributions}

We have presented a domain-independent system for definite description interpretation whose development was based on an empirical study of definite description use that included multiannotator experiments. Our system not only attempts to find an antecedent for a definite description, it also uses methods for recognizing discoursenew descriptions, which our previous studies revealed to be the largest class of definite descriptions in our corpus. Our algorithms for segmentation, matching, and identification of discourse-new descriptions only rely on syntax-based heuristics and on on-line lexical sources such as WordNet; the final configuration of these heuristics, as well as their order of application, was arrived at on the basis of extensive experiments using our training corpus. Because our system only relies on "shallow" information, it encounters problems when commonsense reasoning is actually needed; on the other hand, it can be tested on any domain without extensive handcoding.

As far as direct anaphora is concerned, we evaluated heuristic algorithms for segmentation and matching. Our system achieved $62 \%$ recall and $83 \%$ precision for direct anaphora resolution on our test data. For identifying discourse-new descriptions, we exploited the correlation between certain types of syntactic constructions and type of use noted by Hawkins (1978) and semantically explained by Löbner (1987). Our system achieved $69 \%$ recall and $72 \%$ precision for this class on the test data. Overall, the version of the system that only attempts to recognize first-mention and subsequentmention definite descriptions achieved a recall of $53 \%$ and a precision of $76 \%$ on the test corpus if we count the definite descriptions the system can't handle as errors; if we count them as discourse-new, both recall and precision are $66 \%$.

The class of bridging descriptions is the most difficult to process: this is in part because humans themselves do not agree much on which definites count as bridges and what their anchors are, in part because lexical knowledge and commonsense reasoning are necessary to solve them. Our results for this class are, therefore, still very tentative; this did not much affect the performance of the system, however, since in the texts we tried, bridging descriptions are a relatively small class. Noncoreferent 
bridging descriptions were around $8 \%$ of the definite descriptions in the corpus, and the class of bridging descriptions including those with a coreferent antecedent with a different head noun were about $15 \%$ of the total. We tried techniques that do not involve heavy axiomatization of commonsense knowledge, and only used an existing lexical source, WordNet.

In other text genres the distribution of definite descriptions into classes might change; spoken dialogue, for example, tends to have a higher number of deictic definite descriptions. However, other researchers (Fraurud 1990) found a similar distribution of first-mention and subsequent-mention definites in text corpora; we believe therefore that the heuristics we propose here, and their ordering, will still be adequate. Direct anaphora and discourse-new descriptions can be processed with much simpler methods and it seems that the distinguishing features do not usually overlap.

\subsection{What's Needed Next?}

We would like to emphasize again that we are not trying to suggest that shallow methods will be sufficient for processing definite descriptions in the long run. What we do believe is that hypotheses about processing should be evaluated; unfortunately, only fairly simple techniques can be tested in this way at the moment, but this work can serve to motivate more clearly the use of more complex methods.

We highlighted throughout the paper, and particularly in Section 5, some of the points where shallow methods break down, and better lexical sources or commonsense knowledge are needed. By far the worse results are obtained for bridging descriptions; in this area, the most urgent needs are better sources of lexical knowledge, ${ }^{34}$ and some robust focusing mechanism. Finding better ways of segmenting the text is perhaps the area in which the most progress has been made since we started this project; robust methods for text segmentation are now available (Hearst 1997; Richmond, Smith, and Amitay 1997). A proper treatment of modification seems harder; as discussed in Section 4.1 , it seems necessary to rely heavily on reasoning in some cases. In order to improve our treatment of discourse-new descriptions it will be necessary, on the one hand, to find ways of automatically acquiring lexical information about the functionality of nouns and adjectives, and on the other hand, to have sources of encyclopedic knowledge available.

\subsection{Future Work}

8.3.1 Simple Extensions. In this project we were more interested in clearly identifying the subtasks of the definite description process that in achieving optimum performance; as a consequence, there are a number of fairly simple ways in which the final version of the system could be improved. The next step in making our system truly testable on any type of text would be to make it work off the output of a robust parser: we are currently testing Abney's CASS parser (Abney 1991) for this purpose. See Ishikawa (1998), for some initial results. We are also experimenting with existing software that performs in a more sophisticated way some of the tasks that our system currently implements in a fairly crude fashion, including lemmatization, proper name recognition, and named entity typing.

Another aspect of the system that deserves further examination is the construction of coreference chains and cases of multiple resolutions. We did not get a clear picture

34 As mentioned above, we have done some preliminary work on acquiring this information automatically (Poesio, Schulte im Walde, and Brew 1998; Ishikawa 1998). 
of how complete or incomplete, or how broken, the coreferential chains resulting from the processing of one text are, nor did we relate them to the chains of the annotated texts; to do so, the system and the annotation would have to be extended to cover all cases of anaphoric expressions.

8.3.2 The Role of Focus in Definite Descriptions Processing. Our tests with bridging descriptions resulted in a great number of false positives. Our analysis of these data, as well as of other corpora (Hitzeman and Poesio 1998), suggests that a local focusing mechanism as proposed in Grosz (1977), Sidner (1979), Grosz, Joshi, and Weinstein (1983, 1995), and Grosz and Sidner (1986) would improve the results obtained by our system.

There are several reasons why our system does not yet include such a mechanism. One problem already mentioned is that Sidner's algorithms as stated, and even as implemented by Carter, are difficult to implement, since considerably more lexical information is needed than we have available (e.g., about the thematic roles of verbs), a rich knowledge base is needed both to resolve bridging descriptions and larger situation uses, and commonsense inference is needed to evaluate the plausibility of hypotheses. A second problem with Sidner's theory of local focus, as well as others such as Centering Theory (Grosz, Joshi, and Weinstein 1995), is the lack of a precise characterization of how to deal with complex sentences. Revisions and extensions of Sidner's proposal related to these problems have been proposed in Suri and McCoy (1994), and include algorithms for updating focus in complex sentences containing adjunct clauses such as before- and after-clauses.

We plan to incorporate simpler focus-tracking mechanisms in future versions of the system, possibly along the lines of Azzam, Humphreys, and Gaizauskas (1998) or Tetreault (1999).

8.3.3 Theoretical Developments. We defended the importance of developing methods for identifying discourse-new descriptions, and we believe that there is still need for research into the semantics of this class; that is, what, exactly, licenses the use of a definite description to refer to a discourse-new entity? The role of premodification and postmodification should also be further examined. Postmodification is one of the most frequent features of discourse-new descriptions; additional empirical studies considering a detailed subclassification of discourse-new descriptions would give us a better understanding of the problem. The postmodification of a description often acts as an explicit anchor (what Löbner [1987] calls "disambiguating arguments and attributes"); understanding how the head noun of a postmodified description relates "semantically" with its complement is a problem similar to that of identifying the semantic relation between a bridging description and its anaphoric anchor, but to date there hasn't been much research on this topic (while there has been a lot of work on identifying the relations that hold between the premodifiers, especially in noun-noun compounds). An NP's head noun may also corefer with its complement, as seen in the examples in (59):
a. the dream of home ownership
b. the issue of student grants

We also observed that definite descriptions with premodification were responsible for considerable disagreement among the annotators, the reasons for which are still to be explained. 
We wish to thank Ellen Bard, Rafael Bordini, Jean Carletta, Miriam Eckert, Kari Fraurud, Rob Gaizauskas, Janet Hitzeman, Chris Mellish, and our anonymous reviewers for comments, help, and suggestions. Renata Vieira was supported in part by a fellowship from $\mathrm{CNPq}$, Brazil; Massimo Poesio is supported by an EPSRC Advanced Rescarch Fellowship.

\section{References}

Abney, Steve. 1991. Parsing by chunks. In R. Berwick, S. Abney, and C. Tenny, editors, Principle-based Parsing. Kluwer, Dordrecht, pages 257-278.

Alshawi, Hiyan. 1987. Memory and Context for Language Interpretation. Cambridge University Press, Cambridge.

Alshawi, Hiyan. 1990. Resolving quasi-logical forms. Computational Linguistics, 16(3):133-144.

Alshawi, Hiyan, editor. 1992. The Core Language Engine. MIT Press, Cambridge, MA.

Aone, Chinatsu and Scott W. Bennett. 1995. Automated acquisition of anaphora resolution strategies. In Proceedings of the AAAI Spring Symposium on Empirical Methods in Discourse Interpretation and Generation, pages 1-7, Stanford.

Appelt, Douglas, Jerry R. Hobbs, John Bear, David Israel, Megumi Kameyama, Andy Kehler, David Martin, Karen Myers, and Mabry Tyson. 1995. SRI International FASTUS system MUC- 6 test results and analysis. In Proc. of the Sixth Message Understanding Conference, pages 237-248, Columbia, MD, November.

Azzam, Saliha, Kevin Humphreys, and Robert Gaizauskas. 1998. Evaluating a focus-based approach to anaphora resolution. In COLING-ACL' 98 : 36th Annual Meeting of the Association for Computational Linguistics and the 17th International Conference on Computational Linguistics, pages 74-78, Montreal, Quebec, Canada.

Bean, David L. and Ellen Riloff. 1999. Corpus-based identification of non-anaphoric noun phrases. In Proceedings of the 37th Annual Meeting, pages 373-380, University of Maryland. Association for Computational Linguistics.

Bikel, Daniel, Scott Miller, Richard Schwartz, and Ralph Weischedel. 1997. Nymble: A high-performance learning name finder. In Proceedings of the 5th Conference on Applied Natural Language Processing, pages 194-201, Washington,
DC Association for Computational Linguistics.

Bosch, Peter and Bart Geurts. 1989. Processing definite NPs. IWBS Report 78, IBM Germany, July.

Burger, John D. and Dennis Connolly. 1992. Probabilistic resolution of anaphoric reference. In Proceedings of the AAAI Fall Symposium on Probabilistic Approaches to Natural Language, pages 17-24, Cambridge, MA.

Carbonell, Jamie and Ralf D. Brown, 1988. Anaphora resolution: A multi-strategy approach. In Proceedings of the 12th International Conference on Computational Linguistics (COLING-88), pages 96-101, Budapest, Hungary.

Carletta, Jean. 1996. Assessing agreement on classification tasks: The kappa statistic. Computational Linguistics, 22(2):249-254.

Carletta, Jean, Amy Isard, Stephen Isard, Jacqueline C. Kowtko, Gwyneth Doherty-Sneddon, and Anne $\mathrm{H}$. Anderson. 1997. The reliability of a dialogue structure coding scheme. Computational Linguistics, 23(1):13-32.

Carter, David M. 1987. Interpreting Anaphors in Natural Language Texts. Ellis Horwood, Chichester, UK.

Chinchor, Nancy A. 1995. Statistical significance of MUC-6 results. In Proceedings of the Sixth Message Understanding Conference (MUC-6), pages 39-44, Columbia, MD, November 6-8.

Chinchor, Nancy A. 1997. Overview of MUC-7/MET-2. In Proceedings of the Seventh Message Understanding Conference (MUC-7). Available at http://www.muc. saic.com/proceedings/ muc_7_proceedings/overview. html.

Cho, Sehyeong and Anthony S. Maida. 1992. Using a Bayesian framework to identify the referents of definite descriptions. In Proceedings of the AAAI Fall Symposium on Probabilistic Approaches to Natural Language, pages 39-46, Cambridge, MA.

Clark, Herbert H. 1977. Inferences in comprehension. In D. Laberge and S. J. Samuels, editors, Basic Process in Reading: Perception and Comprehension. Lawrence Erlbaum, pages 243-263.

Clark, Herbert $\mathrm{H}$. and Catherine R. Marshall. 1981. Definite reference and mutual knowledge. In A. Joshi, B. Webber, and I. Sag, editors, Elements of Discourse Understanding. Cambridge University Press, New York.

Fellbaum, Christiane, editor. 1998. WordNet: An Electronic Lexical Database. MIT Press, Cambridge, MA. 
Fox, Barbara A. 1987. Discourse Structure and Anaphora. Cambridge University Press, Cambridge, UK.

Fraurud, Keri. 1990. Definiteness and the processing of NPs in natural discourse. Journal of Semantics, 7:395-433.

Gaizauskas, Robert, Takahiro Wakao, Kevin Humphreys, Hamish Cunningham, and Yorick Wilks. 1995. University of Sheffield: Description of the LaSIE System as used for MUC-6. In Proceedings of the Sixth Message Understanding Conference (MUC-6), pages 207-220. Morgan Kaufmann.

Grosz, Barbara J. 1977. The Representation and Use of Focus in Dialogue Understanding. Ph.D. thesis, Stanford University.

Grosz, Barbara J., Aravind K. Joshi, and Scott Weinstein. 1983. Providing a unified account of definite noun phrases in discourse. In Proceedings of the 21st Annual Meeting, pages 44-50. Association for Computational Linguistics.

Grosz, Barbara. J., Aravind. K. Joshi, and Scott Weinstein. 1995. Centering: A framework for modeling the local coherence of discourse. Computational Linguistics, 21(2):202-225. (The paper originally appeared as an unpublished manuscript in 1986.).

Grosz, Barbara J. and Candace L. Sidner. 1986. Attention, intention, and the structure of discourse. Computational Linguistics, 12(3):175-204.

Hahn, Udo; Michael Strube, and Katja Markert. 1996. Bridging textual ellipsis. In COLING '96: Proceedings of the 16th International Conference on Computational Linguistics, pages 496-501, Kopenhagen, Aug 5-9 1996.

Hardt, Daniel. 1997. An empirical approach to VP ellipsis. Computational Linguistics, 23(4):525-541.

Hatzivassiloglou, Vasileios and Kathleen McKeown. 1993. Towards the automatic identification of adjectival scales: clustering adjectives according to meaning. In Proceedings of the 31st Annual Meeting, pages 172-182, Ohio State University. Association for Computational Linguistics.

Hawkins, John A. 1978. Definiteness and Indefiniteness. Croom Helm, London.

Hearst, Marti A. 1997. TextTiling: Segmenting text into multi-paragraph subtopic passages. Computational Linguistics, 23(1):33-64.

Heim, Irene. 1982. The Semantics of Definite and Indefinite Noun Phrases. Ph.D. thesis, University of Massachusetts at Amherst. Hitzeman, Janet and Massimo Poesio. 1998.
Long-distance pronominalisation and global focus. In COLING/ACL'98: 36th Annual Meeting of the Association for Computational Linguistics and 17 th International Conference on Computational Linguistics. Volume 1, pages 550-556, Montreal, Quebec, Canada.

Hobbs, Jerry R., Mark E. Stickel, Douglas A. Appelt, and Paul Martin. 1993.

Interpretation as abduction. Artificial Intelligence Journal, 63:69-142.

Humphreys, Kevin, Robert Gaizauskas, Saliha Azzam, Chris Huyck, B. Mitchell, and Hamish Cunningham. 1998.

University of Sheffield: Description of the LaSIE-II System as used for MUC-7. In Proceedings of the Seventh Message Understanding Conference (MUC-7). Available on the Web at www.muc.saic.com.

lshikawa, Tomonori. 1998. Acquisition of associative information and resolution of bridging descriptions. Master's thesis, University of Edinburgh, Department of Linguistics, Edinburgh, Scotland.

Kameyama, Megumi. 1997. Recognizing referential links: An information extraction perspective. In Proceedings of the ACL Workshop on Operational Factors in Practical, Robust Anaphora Resolution for Unrestricted Texts, pages 46-53, Madrid, Spain, July. Association for Computational Linguistics.

Krippendorff, Klaus. 1980. Content Analysis: An Introduction to its Methodology. Sage Publications, Beverly Hills, London.

Landis, J. R., and G. G. Koch. 1977. The measurement of observer agreement for categorial data. Biometrics, 36:159-174.

Lappin, Shalom and H. J. Leass. 1994. An algorithm for pronominal anaphora resolution. Computational Linguistics, 20(4):535-562.

Lascarides, Alex and Nicholas Asher. 1993. Temporal interpretation, discourse relations and commonsense entailment. Linguistics and Philosophy, 16(5):437-493.

Löbner, Sebastian. 1987. Natural language and generalised quantifier theory. In P. Gärdenfors, editor, Generalized Quantifiers. D. Reidel, Dordrecht, The Netherlands, pages 93-108.

Mani, Inderjeet and T. Richard MacMillan. 1996. Identifying unknown proper names in newswire text. In Bran Boguraev and James Pustejovsky, editors, Corpus Processing for Lexical Acquisition. MIT Press, Cambridge, MA, pages 41-59. Marcu, Daniel. 1999. A decision-based approach to rhetorical parsing. In Proceedings of the 37th Annual Meeting, 
pages 365-372, University of Maryland, June. Association for Computational Linguistics.

Marcus, Mitchell P., Beatrice Santorini, and Mary Ann Marcinkiewicz. 1993. Building a large annotated corpus of English: The Penn Treebank. Computational Linguistics, 19(2):313-330.

McDonald, David. 1996. Internal and external evidence in the identification and semantic categorization of proper names. In Bran Boguraev and James Pustejovsky, editors, Corpus Processing for Lexical Acquisition. MIT Press, Cambridge, MA, pages $21-39$.

Mikheev, Andrei, Marc Moens, and Claire Grover. 1999. Named entity recognition without gazetteers. In Proceedings of EACL, pages 1-8, Bergen, Norway. EACL.

Mitkov, Ruslan. 2000. Towards more comprehensive evaluation in anaphora resolution. In Proceedings of the 2 nd International Conference on Language Resources and Evaluation, pages 1,309-1,314, Athens.

Paik, Woojin, Elizabeth D. Liddy, Edmund Yu, and Mary McKenna. 1996. Categorizing and standardizing proper nouns for efficient information retrieval. In Bran Boguraev and James Pustejovsky, editors, Corpus Processing for Lexical Acquisition. MIT Press, Cambridge, MA, pages $61-73$.

Palmer, David D. and David S. Day. 1997. A statistical profile of the named entity task. In Proceedings of the 5th Conference on Applied Natural Language Processing, pages 190-193, Washington, DC, March. Association for Computational Linguistics.

Poesio, Massimo. 1993. A situation-theoretic formalization of definite description interpretation in plan elaboration dialogues. In Peter Aczel, David Israel, Yasuhiro Katagiri, and Stanley Peters, editors, Situation Theory and its Applications, Volume 3. CSLI, Stanford, chapter 12, pages 339-374.

Poesio, Massimo, Sabine Schulte im Walde, and Chris Brew. 1998. Lexical clustering and definite description interpretation. In Proceedings of the AAAI Spring Symposium on Learning for Discourse, pages 82-89, Stanford, CA, March. AAAI.

Poesio, Massimo and Renata Vieira. 1998. A corpus-based investigation of definite description use. Computational Linguistics, 24(2):183-216.

Poesio, Massimo, Renata Vieira, and Simone Teufel. 1997. Resolving bridging references in unrestricted text. In R. Mitkov, editor, Proceedings of the ACL Workshop on Operational Factors in Robust Anaphora Resolution, pages 1-6, Madrid. Also available as HCRC Research Paper HCRC/RP-87, University of Edinburgh.

Postal, Paul M. 1969. Anaphoric islands. In R. I. Binnick et al., editor, Papers from the Fifth Regional Meeting of the Chicago Linguistic Society, pages 205-235. University of Chicago.

Prince, Ellen F. 1981. Toward a taxonomy of given-new information. In Peter Cole, editor, Radical Pragmatics. Academic Press, New York, pages 223-256.

Prince, Ellen F. 1992. The ZPG letter: Subjects, definiteness, and information status. In S. Thompson and W. Mann, editors, Discourse Description: Diverse Analyses of a Fund-Raising Text. John Benjamins, pages 295-325.

Quinlan, J. Ross. 1993. C4.5: Programs for Machine Learning. Morgan Kaufmann, San Mateo, CA.

Quirk, Randolph, Sydney Greenbaum, Geoffrey Leech, and Jan Svartvik. 1985. A Comprehensive Grammar of the English Language. Longman, London.

Reichman, Rachel. 1985. Getting Computers to Talk Like You and Me. MIT Press, Cambridge, MA.

Richmond, Kevin, Andrew Smith, and Einat Amitay. 1997. Detecting subject boundaries within text: $\mathrm{A}$ language-independent statistical approach. In Proceedings of The Second Conference on Empirical Methods in Natural Language Processing (EMNLP-2), pages $47-54$, Brown University.

Russell, Bertrand. 1905. On denoting. Mind, 14:479-493. Reprinted in Logic and Knowledge, R. C. Marsh, editor, George Allen and Unwin, London.

Sidner, Candace L. 1979. Towards a computational theory of definite anaphora comprehension in English discourse. Ph.D. thesis, MIT.

Siegel, Sydney and N. John Castellan. 1988. Nonparametric statistics for the Behavioral Sciences. 2nd edition. McGraw-Hill.

Strand, Kjetil. 1996. A taxonomy of linking relations. Manuscript. A preliminary version presented at the Workshop on Indirect Anaphora, Lancaster University, 1996.

Sundheim, Beth M. 1995. Overview of the results of the MUC- 6 evaluation. In Proceedings of the Sixth Message Understanding Conference (MUC-6), pages 13-31, Columbia, MD, November 6-8. 
Suri, Linda Z. and Kathleen F. McCoy. 1994. RAFT/RAPR and centering: A comparison and discussion of problems related to processing complex sentences. Computational Linguistics, 20(2):301-317.

Tetreault, Joel R. 1999. Analysis of syntax-based pronoun resolution methods. In Proceedings of the 37th Annual Meeting, pages 602-605, University of Maryland, June. Association for Computational Linguistics.

Vicira, Renata. 1998. Definite Description Resolution in Unrestricted Texts. Ph.D. thesis, University of Edinburgh, Centre for Cognitive Science, February.

Vieira, Renata and Massimo Poesio. 1996. Processing definite descriptions in corpora. Presented at the Discourse Anaphora and Resolution Colloquium (DAARC), Lancaster University, Lancaster, UK. Also available as Research Paper HCRC/RP-86, University of Edinburgh, Human Communication Research Centre.

Vieira, Renata and Simone Teufel. 1997. Towards resolution of bridging descriptions. In Proceedings of the 35th Joint Meeting of the Association for Computational Linguistics, pages 522-524, Madrid.

Vilain, Marc, John Burger, John Aberdeen, Dennis Connolly, and Lynette Hirschman. 1995. A model-theoretic coreference scoring scheme. In Proc. of the Sixth Message Understanding Conference, pages 45-52.

Wacholder, Nina and Yael. Ravin. 1997. Disambiguation of proper names in text. In Proceedings of the 5 th Conference on Applied Natural Language Processing, pages 202-208, Washington, DC, March. Association for Computational Linguistics.

Ward, Gregory, Richard Sproat, and Gail McKoon. 1991. A pragmatic analysis of so-called anaphoric islands. Language, 67:439-474.

Webber, Bonnie L. 1979. A Formal Approach to Discourse Anaphora. Garland, New York.

Wilks, Yorick A. 1975. A preferential pattern-matching semantics for natural language. Artificial Intelligence, 6:53-74. 
